\title{
Identification of the hub genes in gastric cancer through weighted gene co-expression network analysis
}

\author{
Chunyang $\mathbf{L i}^{1,2}$, Haopeng $\mathbf{Y u}^{1,2}$, Yajing Sun ${ }^{1,2}$, Xiaoxi Zeng ${ }^{\text {Corresp., 1, }}$, Wei Zhang ${ }^{1,2}$ \\ ${ }^{1}$ West China Biomedical Big Data Center, West China Hospital, Sichuan University, Cheng, China \\ 2 Medical Big Data Center, Sichuan University, Chengdu, China \\ Corresponding Author: Xiaoxi Zeng \\ Email address: zengxiaoxi@wchscu.cn
}

Background: Gastric cancer is one of the most lethal tumors and is characterized by poor prognosis and lack of effective diagnostic or therapeutic biomarkers. The aim of this study was to find hub genes serving as biomarkers in gastric cancer diagnosis and therapy.

Methods: GSE66229 from Gene Expression Omnibus (GEO) was used as training set. Genes bearing the top $25 \%$ standard deviations among all the samples in training set were performed to systematic weighted gene co-expression network analysis (WGCNA) to find candidate genes. Then, hub genes were further screened by using the "least absolute shrinkage and selection operator" (LASSO) logistic regression. Finally, hub genes were validated in GS54129 dataset from GEO by supervised learning method artificial neural network (ANN) algorithm.

Results: 12 modules with strong preservation were identified by using WGCNA methods in training set. Of which, five modules significantly related to gastric cancer were selected as clinically significant modules, and 713 candidate genes were identified from these four modules. Then, ADIPOQ, ARHGAP39, ATAD3A, C1orf95, CWH43, GRIK3, INHBA, RDH12, SCNN1G, SIGLEC11 and LYVE1 were screened as the hub genes. These hub genes successfully differentiated the tumor samples from the healthy tissues in an independent testing set through artificial neural network algorithm with the area under the receiver operating characteristic curve at 0.946 .

Conclusions: These hub genes bearing diagnostic and therapeutic values, and our results may provide a novel prospect for the diagnosis and treatment of gastric cancer in the future. 
1

2

3

4

5

6

7

8

9

10

11

12

13

14

15

16

17

18

19

20

Correspondence and requests for materials should be addressed to Xiaoxi Zeng. (email: zengxiaoxi@wchscu.cn)

\section{Identification of the hub genes in gastric cancer through} weighted gene co-expression network analysis

$$
\text { Chunyang } \mathrm{Li}^{1,2} \text {, Haopeng } \mathrm{Yu}^{1,2} \text {, Yajing Sun }{ }^{1,2} \text {, Xiaoxi Zeng }{ }^{1,2, *}
$$

Wei Zhang ${ }^{1,2}$

1 West China Biomedical Big Data Center, West China Hospital, Sichuan University, Chengdu, China

2 Medical Big Data Center, Sichuan University, Chengdu, China

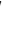

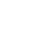

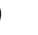

0

1

13

21 Abstract:

Peer) reviewing PDF | (2020:08:51875:2:1:NEW 19 Nov 2020) 
22 Background: Gastric cancer is one of the most lethal tumors and is characterized by poor

23 prognosis and lack of effective diagnostic or therapeutic biomarkers. The aim of this study was

24 to find hub genes serving as biomarkers in gastric cancer diagnosis and therapy.

25 Methods: GSE66229 from Gene Expression Omnibus (GEO) was used as training set. Genes

26 bearing the top $25 \%$ standard deviations among all the samples in training set were performed to

27 systematic weighted gene co-expression network analysis (WGCNA) to find candidate genes.

28 Then, hub genes were further screened by using the "least absolute shrinkage and selection

29 operator" (LASSO) logistic regression. Finally, hub genes were validated in GS54129 dataset

30 from GEO by supervised learning method artificial neural network (ANN) algorithm.

31 Results: 12 modules with strong preservation were identified by using WGCNA methods in

32 training set. Of which, five modules significantly related to gastric cancer were selected as

33 clinically significant modules, and 713 candidate genes were identified from these four modules.

34 Then, ADIPOQ, ARHGAP39, ATAD3A, C1orf95, CWH43, GRIK3, INHBA, RDH12, SCNN1G,

35 SIGLEC11 and LYVE1 were screened as the hub genes. These hub genes successfully

36 differentiated the tumor samples from the healthy tissues in an independent testing set through

37 artificial neural network algorithm with the area under the receiver operating characteristic curve

38 at 0.946 .

39 Conclusions: These hub genes bearing diagnostic and therapeutic values, and our results may

40 provide a novel prospect for the diagnosis and treatment of gastric cancer in the future.

41 Keywords: gastric cancer, weighted gene co-expression network analysis, WGCNA, LASSO

42 regression, supervised machine learning. 
44

45

46

47

48

49

50

51

52

53

54

55

56

57

58

59

60

61

62

63

\section{Background}

Gastric carcinoma remains the fifth most frequently diagnosed cancer and the third leading cause of cancer-related deaths, with an estimated 1,033,701 new cases and 782,685 deaths worldwide in 2018 (Bray et al. 2018; Pormohammad et al. 2018). Gastric cancer is also one of the most common malignancies and the third leading cause of death in China, where 427,100 cases with 301,200 deaths were observed in 2013 (Chen et al. 2017b). Despite the several existing treatments including chemo-, radio-, or targeted therapy, the overall 5-year survival rate of stomach cancer patients is still $<20 \%$ (Raimondi et al. 2018).

There are two types of gastric cancer, diffuse and intestinal types, which differ in their histological manifestations, epidemiological features and etiologic pathogenesis (Huang et al. 2019). Histopathology is the gold standard approach for diagnosing gastric cancer; however, this approach is not suitable for everyone due to the invasive nature of the biopsy (Yoon \& Kim 2015). Although there are several commonly used serum biomarkers such as alpha-fetoprotein (AFP), carcinoembryonic antigen (CEA), cancer antigen 125 (CA125), and cancer antigen 19-9

(CA19-9) (He et al. 2013) for gastric cancer diagnosis, none of them are sensitive or gastriccancer-specific (Smyth et al. 2016). Moreover, effective and specific targeted therapies for gastric cancer remain to be identified. Presently, the major treatment strategies for gastric cancer are anti-human epidermal growth factor receptor 2 (HER2) and anti-vascular therapies (Raimondi et al. 2018). However, resistance to the targeted agents is common in some gastric 
64 tumor types. Therefore, novel practical approaches are needed for specific diagnosis and

65 effective treatment of gastric cancer. Accordingly, identification of the key genes and biomarkers

66 that are involved in the pathogenesis of gastric cancer is of paramount significance.

68 With recent advancements in bioinformatics methods, comprehensive identification of potential

69 biomarkers through large-scale screening of expression profiles has been proposed (Li et al.

70 2018a; Takeno et al. 2008; Wang et al. 2014; Zeng et al. 2019). A weighted gene co-expression

71 network analysis (WGCNA) approach provides a systematic analysis to investigate the

72 functional clustering of expression profiles, based on the theory that genes with similar

73 expression profiles may have closely functional linkages and/or pathways (Carlson et al. 2006;

74 Carter et al. 2004; Zhou et al. 2018). This approach groups highly co-expressed genes into the

75 same module. Modules bearing high correlation with certain clinical traits are identified as

76 clinically significant modules (Zhou et al. 2018).

78 By using this systematic bioinformatic method, followed by the "least absolute shrinkage and

79 selection operator" (LASSO) logistic regression, a suitable method for high-dimensional gene

80 data analysis (Friedman et al. 2010; Zeng et al. 2019), candidate variables were selected from

81 clinically significant modules. Finally, supervised artificial neural network (ANN) method was

82 performed to test the reliability of the results in an independent dataset. ANN approach has been

83 widely used in the prediction of cancer diagnosis, staging and recurrence since the mid-1990s 
84 (Hu et al. 2013), which is an useful method to incorporate and analyze large amounts of omics

85 and health-care data (Ngiam \& Khor 2019).

87 Consequently, we attempt to construct a co-expression network by using systematic WGCNA

88 method followed by LASSO regression to identify hub genes, which could effectively

89 discriminate cancer samples from normal tissue. These findings may provide potential diagnostic

90 and therapeutic targets in future research and clinical intervention of gastric cancer.

92 Materials and methods

93 Data collection and preprocessing

94 The workflow of this study is shown in Figure 1. Raw expression datasets were downloaded

95 from the Gene Expression Omnibus (GEO) database (http://www.ncbi.nlm.nih.gov/geo/) by

96 using the keywords "stomach/gastric cancer/tumor/carcinoma," "normal," "GPL570," and

97 "Homo sapiens." Our inclusion criteria for the training set were that: 1) datasets based on the

98 Affymetrix Human Genome U133 Plus 2.0 Array Platform (Affymetrix, Santa Clara, CA, USA)

99 and 2) datasets derived from human case-control studies, with gastric tumor patients as the case

100 group, regardless of the histopathological types and stages, and non-tumor individuals as the

101 control group.

102

103 Therefore, only two datasets [GSE66229 (Cristescu et al. 2015; Oh et al. 2018) and GSE54129]

104 as of October 10, 2020 met the screening criteria. GSE66229 contained 300 tumor and 100 
105 normal samples, and was used as the training set to screen for the hub genes. GSE54129

106 (contained 111 tumor and 21 normal samples) served as an independent testing set to validate the

107 hub genes.

108

109 All the analyses in this study were conducted using R software (version 3.5.1). FitPLM weight,

110 Relative Log Expression (RLE), Normalized Unscaled Standard Errors (NUSE), and RNA

111 degradation images were analyzed to evaluate the quality of each dataset. Then, the "rma"

112 function with the default parameters of the "affy" package was used to perform background

113 correction and normalization (Gautier et al. 2004). Missing values in each dataset were imputed

114 by using the function "impute.knn" with the default parameters of the "impute" package (Hastie

115 T 2001). Platform annotations were downloaded from the GEO database, and finally, the gene

116 symbol expression matrices were acquired from each dataset for further analyses.

\section{Weighted gene co-expression network construction}

119 Weighted gene co-expression network in the training set was constructed using the "WGCNA"

120 package (Langfelder \& Horvath 2008; Zhang \& Horvath 2005). The genes with the top 25\% SD

121 among all the 400 samples in the expression matrix of the training set were selected as the input

122 genes (5115 genes in total).

123 In brief, first, the appropriate soft-thresholding power $(\beta)$ was selected by using the

124 "pickSoftThreshold" function with the default parameters (herein, $\beta=4$ ). Subsequently, the

125 Pearson's correlation matrix was calculated to evaluate the similarity among all the pair-wise 
126 genes by using the "cor" function with the default parameters. Then, the adjacency was

127 calculated based on $\beta$ and the Pearson's correlation matrix by using the "TOMsimilarity"

128 function with the default parameters, and the corresponding dissimilarity (dissTOM) was also

129 calculated. Finally, average linkage hierarchical clustering was conducted according to the

130 dissTOM value with a minimum size of 30 for each gene dendrogram.

131

132 Module eigengenes (MEs), considered the first principal component (PC) of gene expression

133 patterns of a corresponding module, were obtained for each module. To further strengthen the

134 reliability of the modules, a cut line was set at 0.25 so that modules bearing $<0.25$ would be

135 merged (Chen et al. 2017a).

136

137 Module preservation analysis

138 To evaluate the stability of the modules in the training set, GSE13911 was used to validate the

139 module preservation of the training set (Chen et al. 2018; Neidlin et al. 2019; Obeidat et al.

140 2017). Preservation analysis for GSE13911 was performed using the "modulePreservation"

141 function by setting referenceNetworks $=2$, nPermutations $=200$, randomSeed $=1$, and verbose $=$

1423 , maxModuleSize $=3000$ and maxGoldModuleSize $=3000$ in "WGCNA" package. The $Z$

143 summary scores of each module were calculated to indicate the module preservations. $Z$

144 summary scores $<2$, [2-10], and $>10$ indicated that the modules had no, moderate, and strong

145 preservation, respectively (Liu et al. 2019b; Lou et al. 2017). The grey module contained the

146 genes that did not belong to any of the other modules, and a gold module was generated for 
147 statistical purposes. Therefore, these two modules were not shown in the preservation analysis

148 results (Langfelder et al. 2011a).

\section{Identification of clinically significant modules}

151 Herein, the most interesting clinical trait was the tissue type, which was designated as tumor or

152 normal samples. We calculated $\log _{10}$ transformation of the $P$-value in the logistic regression

153 between the MEs and clinical trait. Modules with $\log _{10}$ transformation of the $P$-value greater than

15410 were considered to be closely correlated with tissue types.

\section{Functional enrichment and pathway analyses of significant modules}

157 To determine whether the clinically significant modules were closely correlated with gastric

158 cancer, GO functional annotation and KEGG pathway analyses were performed using the

159 Database for Annotation, Visualization and Integrated Discovery (DAVID) (version 6.8)

160 (https://david.ncifcrf.gov/home.jsp)(Ashburner et al. 2000; Dennis et al. 2003). The visualization

161 of the functional enrichment and pathway analyses was performed by the "GOplot" (Robin et al.

162 2011) and "ggplot2" (H 2016) packages of R, respectively.

163

\section{Candidate gene selection}

165 Candidate genes among clinically significant modules were selected according to the following

166 criteria: 1) differentially expressed genes (DEGs) between gastric cancer samples and normal

167 samples with $\mid \log _{2} \mathrm{FC}$ (Fold Change) $\mid>1$ and adjusted $P$-value $<0.05$ based on the "limma" 
168 package (Ritchie et al. 2015), 2) high module membership (defined as the correlation between

169 the expression of each genes and MEs) $\mid$ MM(Module Membership $) \mid>0.8$.

170

171 Selection of hub genes by LASSO logistic regression analysis

172 Candidate genes were subjected to LASSO regression, which was performed using the "glmnet"

173 package by setting alpha $=1$, and ten-fold cross-validation for tuning parameter selection.

174 Lambda was defined as the minimum partial likelihood deviance (Friedman 2010).

175

\section{Validation of hub genes}

177 The machine learning method of ANN (by using the "neuralnet" function with hidden $=2$ )

178 (Stefan Fritsch 2019) was performed to determine whether the hub genes could correctly

179 distinguish the gastric cancer samples from the normal samples in a testing set. Moreover, in

180 order to demonstrate whether these hub genes could specifically distinguish between gastric

181 tumor and normal samples, we also evaluated the predictive effects of 11-gene model in

182 pancreatic cancer (GSE45436) (Badea et al. 2008) and colorectal cancer (GSE37364 excluding

183 adenoma samples) (Galamb et al. 2012) by using ANN algorithm.

184 Areas under the receiver operating characteristic (ROC) curve was calculated to show the

185 predictive effect of supervised machine learning model, and then the ROC curves were plotted

186 using the "pROC" package (Robin et al. 2011). An area under the curve (AUC) value between

1870.8 and 0.9 is considered an excellent classification, while greater than 0.9 is considered as

188 outstanding discrimination (Lemeshow 2000). 


\section{Results}

\section{Construction of co-expression networks}

192 After the quality check of the input data, no sample was removed (Supplementary Figure 1);

193 herein, two clinical traits (tissue type and stage) are presented. According to different tissue types

194 (tumor or normal), the 400 samples could be mainly divided into two clusters.

196 As shown in Figure 2, the soft thresholding was set at 4, while the scale-free topology fit index

197 reached 0.89, indicating approximate scale-free topology. Co-expressed gene modules were

198 identified with the dynamic tree cut method (Figure 3A) (Chen et al. 2017a). In total, 12

199 modules were found, and each color represented one module (Figure 3B). The biggest module

200 was the turquoise module, which contained 2120 genes, followed by the blue module, bearing

2011503 genes. The grey module comprised 2 genes, which did not have a similar expression pattern

202 and did not belong to any other module.

203

204 Module preservation analysis

2055115 genes in GSE13911 clustered into 11 colored modules (grey module only contained 2

206 genes, and did not show in Figure 4), as determined in the training set. All gene modules were

207 found to bear strong conservation, as the $Z$ summary scores were all $>10$ (Figure 4B).

208

209 Selection of clinically significant modules 
210 After the assessment of the relationship by using regression analysis between the MEs and

211 clinical traits, the $\log _{10}$ transformation of the $P$-value was shown in Figure 5. Accoring to the

212 screening criteria, there were 5 modules closely related to tissue types, which were black,

213 turquoise, greenyellow, salmon and blue modules. These moduels were selected for further

214 analysis.

215

216 Functional enrichment of clinically significant modules

217 Gene Ontology (GO) enrichment results showed that 1503 genes in the blue module, 137 genes

218 in the black module, 2120 genes in the turquoise module, 61 in the salmon module and 194

219 genes in the greenyellow module mainly participated in 141, 15, 221, 54 and 146 different

220 significant biological processes, respectively (Supplementary Table 1 to Supplementary

221 Table 5). The top three most significantly enriched biological processes were cell division $(P=$

222 2.29e-29), G1/S transition of the mitotic cell cycle $(P=6.32 \mathrm{e}-21)$, mitotic nuclear division $(P=$

223 3.49e-19) in the blue module (Figure 6A), and potassium ion import $(P=3.39 \mathrm{e}-05)$, digestion $(P$

$224=8.25 \mathrm{e}-04)$, multicellular organismal water homeostasis $(P=0.0012)$ in the black module

225 (Figure 6B), and extracellular matrix organization $(P=6.30 \mathrm{e}-06)$, positive regulation of cell

226 migration $(P=7.57 \mathrm{e}-06)$, axon guidance in the turquoise module $(P=9.18 \mathrm{e}-06)$ (Figure 6C),

227 and inflammatory response $(P=2.80 \mathrm{e}-21)$, immune response $(P=5.70 \mathrm{e}-17)$, neutrophil

228 chemotaxis $(P=5.15 \mathrm{e}-15)$ in the greenyellow module (Figure 6D), and defense response to

229 virus $(P=3.33 \mathrm{e}-25)$, type $\mathrm{I}$ interferon signaling pathway $(P=8.52 \mathrm{e}-23)$, response to virus $(P=$

$2309.02 \mathrm{e}-16$ ) in salmon module (Figure 6E). 
232 KEGG (Kyoto Encyclopedia of Genes and Genomes) analysis showed that genes in the blue,

233 black, turquoise, salmon and greenyellow modules were mainly significantly enriched in 15, 7,

234 35,14 and 23 pathways, respectively (from Supplementary Table 6 to Supplementary Table

235 10). The top three most significantly enriched pathways were cell cycle $(P=1.21 \mathrm{e}-15)$, DNA

236 replication $(P=6.21 \mathrm{e}-15)$, the p53 signaling pathway $(P=2.03 \mathrm{e}-06)$ in blue module (Figure

237 7A), and gastric acid secretion $(P=4.46 \mathrm{e}-05)$, protein digestion and absorption $(P=1.28 \mathrm{e}-04)$,

238 drug metabolism-cytochrome P450 $(P=0.0032)$ in black moule (Figure 7B), and focal adhesion

$239(P=1.49 \mathrm{e}-07)$, arrhythmogenic right ventricular cardiomyopathy $(P=2.27 \mathrm{e}-05)$, complement

240 and coagulation cascades $(P=3.58 \mathrm{e}-05)$ in turquoise module (Figure 7C), and cytokine-

241 cytokine receptor interaction $(P=2.35 \mathrm{e}-08)$, amoebiasis $(P=3.30 \mathrm{e}-05)$, and rheumatoid arthritis

$242(P=5.65 \mathrm{e}-05)$ in greenyellow module(Figure 7D). While in the salmon module, influenza A $(P$

$243=1.09 \mathrm{e}-09)$, measles $(P=9.44 \mathrm{e}-07)$, and herpes simplex infection $(P=7.99 \mathrm{e}-06)($ Figure $7 \mathbf{E})$

244 were the most significantly enriched pathways.

\section{Identification of hub genes}

247 Using the screening criteria of $|\mathrm{MM}|>0.8,926$ genes were identified from 5 significant modules,

248 of which 713 genes were differentially expressed between the normal and tumor samples with

$249|\log \mathrm{FC}|>1$ and adjusted $P$-value $<0.05$ (all the 713 candidate genes were listed in

250 Supplementary Table 11). Finally, 11 genes [Adiponectin ( $A D I P O Q)$; Rho GTPase activating

251 protein 39 (ARHGAP39); ATPase family AAA-domain containing protein 3A (ATAD3A); 
252 Clorf95 (also known as STUM gene); Cell wall biogenesis 43 C-terminal homolog (CWH43);

253 Glutamate receptor, ionotropic kainate 3 (GRIK3); Inhibin subunit beta A (INHBA); sodium

254 channel epithelial 1 subunit gamma $(S C N N 1 G)$; Sialic acid-binding immunoglobulin-like lectin-

25511 (SIGLEC11); Retinol dehydrogenase 12 (RDH12) and lymphatic vessel endothelial

256 hyaluronan receptor $1(L Y V E 1)$ ] were identified as the hub genes by using LASSO logistic

257 regression (Table 1). The heatmap of these 11 hub genes were shown in Figure 8, indicating that

258 these 11 hub genes differentially expressed between tumor and normal samples.

260 Validation of the hub genes

261 GSE54129 was utilized as the testing set to validate the 11-gene model. The AUC value of this

262 classifier upon using artificial neural network was 0.946 indicating the excellent classification

263 effects of the model (Figure 9A). Furthermore, both the AUC values of this 11-gene model were

264 around 0.5 in colorectal cancer (Figure 9B) and pancreatic cancer (Figure 9C), indicating

265 specifically predictive effect in gastric cancer.

266

267 Discussion

268 In this study, four modules were identified as clinically significant and preserved modules by

269 using WGCNA. The GO and KEGG analyses revealed that the genes in these four modules were

270 significantly enriched in the biological processes of the cell cycle, cell division, and stomach-

271 related functions. All these biological functions are closely related to gastric cancer (Cao et al.

272 2018; Waldum et al. 2017). Eventually, 11 hub genes including ADIPOQ, ARHGAP39, 
273

274

275

276

277

278

279

280

281

282

283

284

285

286

287

288

289

290

291

292

293

ATAD3A, C1orf95, CWH43, GRIK3, INHBA, RDH12, SCNN1G, SIGLEC11 and LYVE1 were

screened by using WGCNA method followed by LASSO regression. Then, artificial neural

network algorithms were performed, and demonstrated that this 11-gene model could effectively

discriminate between gastric cancer and normal tissues.

In preservation analysis, $\mathrm{Z}$ summary is used to assess the significance of observed statistics and

is defined as the mean of $\mathrm{Z}$ scores computed for density and connectivity measures (Lou et al.

2017). When density and connectivity based preservation statistics are important factors for

judging the preservation of a network, $\mathrm{Z}$ summary score was preferentially selected to evaluate

the preservation (Langfelder et al. 2011b). Although turquoise, blue and greenyellow had low

preservation according to the median rank results, they are still considered to be conserved since

the $\mathrm{Z}$ summary scores of these modules were 39,52 and 22, respectively. Black module has

relatively high $\mathrm{Z}$ summary score and low median rank, indicating high preservation. In the

current study, all the four gene modules were considered to be conserved and selected for further

analysis.

INHBA, Inhibin- $\beta$ A (INHBA), a ligand belonging to the transforming growth factor- $\beta$

superfamily (Oshima et al. 2014), is associated with cell proliferation in various tumor types

including colon adenocarcinoma (Lin et al. 2020a; Miao et al. 2020; Miyamoto et al. 2020),

pancreatic cancer (Liu et al. 2020), gastric cancer (Chen et al. 2019) as well as oral squamous

cell carcinoma (Lin et al. 2020b). Many studies have demonstrated the prognostic role of INHBA 
294 in colon adenocarcinoma (Chen et al. 2020; Li et al. 2020; Miao et al. 2020; Sun et al. 2020), and

295 the role of INHBA in gastric cancer has been widely reported also. INHBA was highly expressed

296 (Kaneda et al. 2011; Seeruttun et al. 2019; Zhang et al. 2010) and aberrantly methylated (Zhang

297 et al. 2019) in gastric tumor samples, and high INHBA expression was associated with

298 significantly poorer 5-year survival than low expression group (Katayama et al. 2017; Wang et

299 al. 2012). One study has demonstrated that INHBA gene silencing could inhibit gastric cancer

300 cell migration and invasion by impeding TGF- $\beta$ signaling pathway (Chen et al. 2019).

301

302 ADIPOQ is one of the most important adipocytokines secreted by adipocytes (Parida et al.

303 2019), and the polymorphisms of $A D I P O Q$ have been reported to correlate with several types of

304 cancer, including colorectal (Nimptsch et al. 2017; Tan et al. 2017) and breast (Mendez-

305 Hernandez et al. 2017) cancers. A study focusing on the molecular mechanisms ADIPOQ

306 participated in has revealed that ADIPOQ induces cytotoxic autophagy in breast cancer cells

307 through STK11/LKB1-mediated activation of the AMPK-ULK1 axis (Chung et al. 2017).

308 Another study has reported that miR-370 inhibits the proliferation, invasion, and epithelial-

309 mesenchymal transition of gastric cells by directly downregulating receptor 4 of ADIPOQ (Feng

310 et al. 2018). Overexpression of another microRNA, miR-15b-5p, promotes the metastasis of

311 gastric cancer by regulating ADIPOQ receptor 3 (Zhao et al. 2017).

312

313 ATAD3A is a nuclear-encoded mitochondrial enzyme, involving in mitochondrial dynamics, cell

314 death, and cholesterol metabolism (Teng et al. 2019). It has been reported to correlate with 
315 hepatocellular carcinoma (Liu et al. 2019a) and breast cancer (Daniel et al. 2019), and it might

316 be an effective therapeutic target in cancer treatment (Teng et al. 2016). ATAD3A is differentially

317 expressed between paclitaxel-resistant and -sensitive MCF7 breast cancer cells (Daniel et al.

318 2019). A study has revealed that ATAD3A is upregulated in hepatocellular carcinoma and

319 ATAD3A upregulation is correlated with poor prognosis (Liu et al. 2019a).

320

321 LYVE1 acts as a receptor and binds to both soluble and immobilized hyaluronan (Banerji et al.

322 2016), may function in lymphatic hyaluronan transport and tumor metastasis (Wu et al. 2019).

323 The dysregulation of LYVE1 closely correlated with many types of tumor, like gastric cancer

324 (Ozmen et al. 2011), colorectal cancer (Gao et al. 2006), breast cancer (Kato et al. 2005), lung

325 cancer (Koukourakis et al. 2005) and liver cancer (Mouta Carreira et al. 2001). LYVE-1 have

326 been studied extensively for its possible role in cancer diagnosis and prognosis in cancer. One

327 study has demonstrated that LYVE1 was upregulated in gastric cancer, and overexpression of

328 LYVE1 positively correlated with perineural invasion and lymph node in gastric cancers (Ozmen

329 et al. 2011). And the expression of LYVE1 might be an biomarker to predict the existence of

330 regional lymph node metastasis in early gastric cancer (Fujimoto et al. 2007).

331

332 RDH12, an NADPH-dependent retinal reductase, catalyzes the reduction of all-trans retinal to

333 all-trans retinol (Belyaeva et al. 2005). It was significantly decreased in gastric tumor samples

334 (Kropotova et al. 2013) and cervical squamous cell carcinoma samples (Peng et al. 2015). 
335 RDH12 was also one of the differentially expressed metabolism-related genes, and correlated

336 with the prognosis of gastric cancer patients (Wen et al. 2020).

337

338 GRIK3 mainly participates in the neuroactive ligand-receptor interaction pathway, and GRIK3

339 upregulation is associated with poor survival in gastric cancer (Gong et al. 2017). GRIK3

340 promotes epithelial-mesenchymal transition by regulating the SPDEF/CDH1 signaling in breast

341 cancer cells (Xiao et al. 2019).

342

343 There have been few studies focusing on the relationship between gastric cancer and $S C N N 1 G$,

344 ARHGAP39, Clorf95, CWH43 or SIGLEC11. SCNN1G is one of the genes significantly

345 upregulated in Ewing's sarcoma and fibromatosis samples (Sarver et al. 2015). ARHGAP39

346 mutations or variations in copy number or expression level are found in several types of tumor-

347 like tissues from the central nervous system, skin, prostate, and gastrointestinal tract (Nowak

348 2018). ARHGAP39 interacts with p53 and BAX, and decreased expression

349 of ARHGAP39 increases cell proliferation, leading to tumorigenesis (Jones 2017). Clorf95 is one

350 of the uncharacterized proteins correlated with diverse human cancers (Delgado 2014). Another

351 study focusing on scleroderma patients demonstrated the involvement of Clorf95 in cancer

352 incidence (Xu et al. 2016). Sialic acid-binding immunoglobulin-like lectin-11 (SIGLEC11) is a

353 primate-lineage-specific receptor of human tissue macrophages, and it is also expressed in brain

354 microglia (Angata et al. 2002; Shahraz et al. 2015). A missense mutation of SIGLEC11 has been

355 detected in pancreatic cancer patients (Jones et al. 2008), and SIGLEC11 is significantly 
356 upregulated in the poor prognostic group of pancreatic cancer patients (Stratford et al. 2010).

357 CWH43 was correlated with tumorigenesis in thyrotropin-secreting pituitary adenomas (Sapkota

358 et al. 2017). One meta-analysis also showed that CWH43 was differentially expressed between

359 colorectal cancer and normal (Chu et al. 2014) samples.

360

361 All these results from the previous studies demonstrate that the hub genes identified in our study

362 are closely correlated with gastric cancer and play important roles in cancer development,

363 progression, or proliferation.

364

365 The significant module and hub genes identified in this study are biologically rational. First, the

366 clinically significant module identified in our study bears strong preservation, implying that this

367 clinically significant module is conservative and could also be reproduced in other datasets.

368 Further, it suggests that that modules constructed by WGCNA are reliable. Second, most of the

369 genes in the significant module were enriched for specific GO terms and KEGG pathways

370 closely relating to stomach or cancer physiology. For instance, GO analysis demonstrated that

371 most of the genes in the clinically significant modules were closely related to digestion,

372 carbohydrate metabolic process, and gastric acid secretion, as well as cell division and cell cycle.

373 KEGG enrichment analysis also indicated that most of the genes in the clinically significant

374 module were implicated in gastric acid secretion, protein digestion and absorption, as well as

375 glycerolipid metabolism and the p53 signaling pathway. Third, all the hub genes identified in our

376 study had previously been reported to relate to cancer. Moreover, several hub genes are 
377 implicated in metabolic processes, influencing the development and progression of gastric

378 cancer. A previous study has demonstrated the association between metabolic syndrome and

379 gastric cancer ( $\mathrm{Li}$ et al. 2018b). A study has detected increased fatty acid oxidation in gastric

380 cancer (Lee et al. 2019), and adipocytes fuel gastric cancer by mediating fatty acid metabolism

381 (Tan et al. 2018). It may thus be inferred that these genes are genuinely the hub genes in charge

382 of the key processes in gastric cancer, and they deserve a deeper analysis and validation. Finally,

383 by using machine learning methods, the hub genes were demonstrated to effectively discriminate

384 the gastric tumor samples from normal samples. In our study, the predictive effects of ANN

385 method was evaluated by AUC values (Huang \& Ling 2005). Herein, the AUC value was $>0.8$,

386 indicating the excellent predictive results. Furthermore, these 11-gene model might be the

387 specific predictors for gastric cancer, since the AUC values of this predictive model were less

388 than 0.8 in other tumor types including, colorectal cancer and pancreatic cancer. All the results

389 indicated that the expression profiles of these 11 hub genes have excellent predictive effects

390 when discriminating gastric cancer samples from normal samples.

391

392 However, our study has limitations. First, all the hub genes were identified and validated only

393 through bioinformatics, and further exploration of the biological functions and molecular

394 mechanisms of these hub genes both in vitro and in vivo is required. Second, due to the limited

395 availability of the data, we did not differentiate between intestinal-type and diffuse-type gastric

396 cancers. More data are needed to analyze and identify the hub genes between these two types of

397 gastric cancer and normal samples. 
399 In summary, through WGCNA, we identified 11 hub genes, which might serve as potential

400 diagnostic and/or therapeutic biomarkers for gastric cancer. Profile data mining by

401 bioinformatics analysis is an available method to find potential diagnostic or therapeutic

in which these hub genes are involved are still needed to verify the involvement of these genes in

gastric cancer. Our findings provide a better understanding of the molecular mechanisms and

putative diagnostic or therapeutic biomarkers for gastric cancer.

406

407

408

409

410

411

412

413

414

415

416

417

418

419

420

421

422

423

424

425

426

427

428

\section{References}

Angata T, Kerr SC, Greaves DR, Varki NM, Crocker PR, and Varki A. 2002. Cloning and characterization of human Siglec-11. A recently evolved signaling molecule that can interact with SHP-1 and SHP-2 and is expressed by tissue macrophages, including brain microglia. J Biol Chem 277:24466-24474. 10.1074/jbc.M202833200

Ashburner M, Ball CA, Blake JA, Botstein D, Butler H, Cherry JM, Davis AP, Dolinski K, Dwight SS, Eppig JT, Harris MA, Hill DP, Issel-Tarver L, Kasarskis A, Lewis S, Matese JC, Richardson JE, Ringwald M, Rubin GM, and Sherlock G. 2000. Gene ontology: tool for the unification of biology. The Gene Ontology Consortium. Nat Genet 25:25-29. 10.1038/75556

Badea L, Herlea V, Dima SO, Dumitrascu T, and Popescu I. 2008. Combined gene expression analysis of wholetissue and microdissected pancreatic ductal adenocarcinoma identifies genes specifically overexpressed in tumor epithelia. Hepatogastroenterology 55:2016-2027.

Banerji S, Lawrance W, Metcalfe C, Briggs DC, Yamauchi A, Dushek O, van der Merwe PA, Day AJ, and Jackson DG. 2016. Homodimerization of the Lymph Vessel Endothelial Receptor LYVE-1 through a Redox-labile Disulfide Is Critical for Hyaluronan Binding in Lymphatic Endothelium. J Biol Chem 291:25004-25018. 10.1074/jbc.M116.736926

Belyaeva OV, Korkina OV, Stetsenko AV, Kim T, Nelson PS, and Kedishvili NY. 2005. Biochemical properties of purified human retinol dehydrogenase 12 (RDH12): catalytic efficiency toward retinoids and C9 aldehydes and effects of cellular retinol-binding protein type I (CRBPI) and cellular retinaldehyde-binding protein (CRALBP) on the oxidation and reduction of retinoids. Biochemistry 44:7035-7047. 10.1021/bi050226k

Bray F, Ferlay J, Soerjomataram I, Siegel RL, Torre LA, and Jemal A. 2018. Global Cancer Statistics 2018: GLOBOCAN Estimates of Incidence and Mortality Worldwide for 36 Cancers in 185 Countries. CA Cancer J Clin. 10.3322/caac.21492 
429

430

431

432

433

434

435

436

437

438

439

440

441

442

443

444

445

446

447

448

449

450

451

452

453

454

455

456

457

458

459

460

461

462

463

464

465

466

467

468

469

Cao J, Wu N, Han Y, Hou Q, Zhao Y, Pan Y, Xie X, and Chen F. 2018. DDX21 promotes gastric cancer proliferation by regulating cell cycle. Biochem Biophys Res Commun 505:1189-1194. 10.1016/j.bbrc.2018.10.060

Carlson MR, Zhang B, Fang Z, Mischel PS, Horvath S, and Nelson SF. 2006. Gene connectivity, function, and sequence conservation: predictions from modular yeast co-expression networks. BMC Genomics 7:40. 10.1186/1471-2164-7-40

Carter SL, Brechbuhler CM, Griffin M, and Bond AT. 2004. Gene co-expression network topology provides a framework for molecular characterization of cellular state. Bioinformatics 20:2242-2250. 10.1093/bioinformatics/bth234

Chen L, Yuan L, Qian K, Qian G, Zhu Y, Wu CL, Dan HC, Xiao Y, and Wang X. 2018. Identification of Biomarkers Associated With Pathological Stage and Prognosis of Clear Cell Renal Cell Carcinoma by Co-expression Network Analysis. Front Physiol 9:399. 10.3389/fphys.2018.00399

Chen L, Yuan L, Wang Y, Wang G, Zhu Y, Cao R, Qian G, Xie C, Liu X, Xiao Y, and Wang X. 2017a. Co-expression network analysis identified FCER1G in association with progression and prognosis in human clear cell renal cell carcinoma. Int J Biol Sci 13:1361-1372. 10.7150/ijbs.21657

Chen S, Cao GD, Wei W, Yida L, Xiaobo H, Lei Y, Ke C, Chen B, and Xiong MM. 2020. Prediction and identification of immune genes related to the prognosis of patients with colon adenocarcinoma and its mechanisms. World J Surg Oncol 18:146. 10.1186/s12957-020-01921-9

Chen W, Zheng R, Zhang S, Zeng H, Xia C, Zuo T, Yang Z, Zou X, and He J. 2017b. Cancer incidence and mortality in China, 2013. Cancer Lett 401:63-71. 10.1016/j.canlet.2017.04.024

Chen ZL, Qin L, Peng XB, Hu Y, and Liu B. 2019. INHBA gene silencing inhibits gastric cancer cell migration and invasion by impeding activation of the TGF-beta signaling pathway. J Cell Physiol 234:18065-18074. 10.1002/jcp.28439

Chu CM, Yao CT, Chang YT, Chou HL, Chou YC, Chen KH, Terng HJ, Huang CS, Lee CC, Su SL, Liu YC, Lin FG, Wetter T, and Chang CW. 2014. Gene expression profiling of colorectal tumors and normal mucosa by microarrays meta-analysis using prediction analysis of microarray, artificial neural network, classification, and regression trees. Dis Markers 2014:634123. 10.1155/2014/634123

Chung SJ, Nagaraju GP, Nagalingam A, Muniraj N, Kuppusamy P, Walker A, Woo J, Gyorffy B, Gabrielson E, Saxena NK, and Sharma D. 2017. ADIPOQ/adiponectin induces cytotoxic autophagy in breast cancer cells through STK11/LKB1-mediated activation of the AMPK-ULK1 axis. Autophagy 13:1386-1403. 10.1080/15548627.2017.1332565

Cristescu R, Lee J, Nebozhyn M, Kim KM, Ting JC, Wong SS, Liu J, Yue YG, Wang J, Yu K, Ye XS, Do IG, Liu S, Gong L, Fu J, Jin JG, Choi MG, Sohn TS, Lee JH, Bae JM, Kim ST, Park SH, Sohn I, Jung SH, Tan P, Chen R, Hardwick J, Kang WK, Ayers M, Hongyue D, Reinhard C, Loboda A, Kim S, and Aggarwal A. 2015. Molecular analysis of gastric cancer identifies subtypes associated with distinct clinical outcomes. Nat Med 21:449-456. 10.1038/nm.3850

Daniel P, Halada P, Jelinek M, Balusikova K, and Kovar J. 2019. Differentially Expressed Mitochondrial Proteins in Human MCF7 Breast Cancer Cells Resistant to Paclitaxel. Int J Mol Sci 20. 10.3390/ijms20122986

Delgado AP, Brandao, P., Chaoado, M.J., Hamid, S., Narayanan, R.,. 2014. Open Reading Frames Associated with Cancer in the Dark Matter of the Human Genome. Cancer Genomics and Proteomics 11:12.

Dennis G, Sherman BT, Hosack DA, Yang J, Gao W, Lane HC, and Lempicki RA. 2003. DAVID: Database for annotation, visualization, and integrated discovery. Genome Biology 4.

Peer] reviewing PDF | (2020:08:51875:2:1:NEW 19 Nov 2020) 
470

471

472

473

474

475

476

477

478

479

480

481

482

483

484

485

486

487

488

489

490

491

492

493

494

495

496

497

498

499

500

501

502

503

504

505

506

507

508

509

510

Feng Y, Sun T, Yu Y, Gao Y, Wang X, and Chen Z. 2018. MicroRNA-370 inhibits the proliferation, invasion and EMT of gastric cancer cells by directly targeting PAQR4. J Pharmacol Sci 138:96-106. 10.1016/j.jphs.2018.08.004

Friedman J. 2010. Package 'glmnet'.

Friedman J, Hastie T, and Tibshirani R. 2010. Regularization Paths for Generalized Linear Models via Coordinate Descent. J Stat Softw 33:1-22.

Fujimoto A, Ishikawa Y, Akishima-Fukasawa Y, Ito K, Akasaka Y, Tamai S, Maehara T, Kiguchi H, Ogata K, Nishimura C, Miki K, and Ishii T. 2007. Significance of lymphatic invasion on regional lymph node metastasis in early gastric cancer using LYVE-1 immunohistochemical analysis. Am J Clin Pathol 127:8288. 10.1309/LJQ9G0X8KP17QXP3

Galamb O, Wichmann B, Sipos F, Spisak S, Krenacs T, Toth K, Leiszter K, Kalmar A, Tulassay Z, and Molnar B. 2012. Dysplasia-carcinoma transition specific transcripts in colonic biopsy samples. PLoS One 7:e48547. 10.1371/journal.pone.0048547

Gao F, Lu YM, Cao ML, Liu YW, He YQ, and Wang Y. 2006. Expression and quantification of LYVE-1 in human colorectal cancer. Clin Exp Med 6:65-71. 10.1007/s10238-006-0097-4

Gautier L, Cope L, Bolstad BM, and Irizarry RA. 2004. affy--analysis of Affymetrix GeneChip data at the probe level. Bioinformatics 20:307-315. 10.1093/bioinformatics/btg405

Gong B, Li Y, Cheng Z, Wang P, Luo L, Huang H, Duan S, and Liu F. 2017. GRIK3: A novel oncogenic protein related to tumor TNM stage, lymph node metastasis, and poor prognosis of GC. Tumour Biol 39:1010428317704364. 10.1177/1010428317704364

H W. 2016. ggplot2: elegant graphics for data analysis.

Hastie T TR, Narasimhan B. 2001. impute: Imputation for microarray data. Bioinformatics 17:6.

He CZ, Zhang KH, Li Q, Liu XH, Hong Y, and Lv NH. 2013. Combined use of AFP, CEA, CA125 and CA19-9 improves the sensitivity for the diagnosis of gastric cancer. BMC Gastroenterol 13:87. 10.1186/1471-230X$13-87$

Hu X, Cammann H, Meyer HA, Miller K, Jung K, and Stephan C. 2013. Artificial neural networks and prostate cancer-tools for diagnosis and management. Nat Rev Urol 10:174-182. 10.1038/nrurol.2013.9

Huang J, and Ling CX. 2005. Using AUC and accuracy in evaluating learning algorithms. Ieee Transactions on Knowledge and Data Engineering 17:299-310. Doi 10.1109/Tkde.2005.50

Huang SC, Ng KF, Yeh TS, Cheng CT, Lin JS, Liu YJ, Chuang HC, and Chen TC. 2019. Subtraction of Epstein-Barr virus and microsatellite instability genotypes from the Lauren histotypes: Combined molecular and histologic subtyping with clinicopathological and prognostic significance validated in a cohort of 1,248 cases. Int J Cancer 145:3218-3230. 10.1002/ijc.32215

Jones R. 2017. Identification of novel risk variants for sarcoma and other cancers by whole exome sequencing in cancer cluster families. PhD Thesis, The University of Western Australia.

Jones S, Zhang X, Parsons DW, Lin JC, Leary RJ, Angenendt P, Mankoo P, Carter H, Kamiyama H, Jimeno A, Hong SM, Fu B, Lin MT, Calhoun ES, Kamiyama M, Walter K, Nikolskaya T, Nikolsky Y, Hartigan J, Smith DR, Hidalgo M, Leach SD, Klein AP, Jaffee EM, Goggins M, Maitra A, Iacobuzio-Donahue C, Eshleman JR, Kern SE, Hruban RH, Karchin R, Papadopoulos N, Parmigiani G, Vogelstein B, Velculescu VE, and Kinzler KW. 2008. Core signaling pathways in human pancreatic cancers revealed by global genomic analyses. Science 321:1801-1806. 10.1126/science.1164368

Peer] reviewing PDF | (2020:08:51875:2:1:NEW 19 Nov 2020) 
511 Kaneda H, Arao T, Matsumoto K, De Velasco MA, Tamura D, Aomatsu K, Kudo K, Sakai K, Nagai T, Fujita Y,

512

513

514

515

516

517

518

519

520

521

522

523

524

525

526

527

528

529

530

531

532

533

534

535

536

537

538

539

540

541

542

543

544

545

546

547

548

549

550

551 Tanaka K, Yanagihara K, Yamada Y, Okamoto I, Nakagawa K, and Nishio K. 2011. Activin A inhibits vascular endothelial cell growth and suppresses tumour angiogenesis in gastric cancer. $\mathrm{Br} J \mathrm{Cancer}$ 105:1210-1217. 10.1038/bjc.2011.348

Katayama Y, Oshima T, Sakamaki K, Aoyama T, Sato T, Masudo K, Shiozawa M, Yoshikawa T, Rino Y, Imada T, and Masuda M. 2017. Clinical Significance of INHBA Gene Expression in Patients with Gastric Cancer who Receive Curative Resection Followed by Adjuvant S-1 Chemotherapy. In Vivo 31:565-571. 10.21873/invivo. 11095

Kato T, Prevo R, Steers G, Roberts H, Leek RD, Kimura T, Kameoka S, Nishikawa T, Kobayashi M, Jackson DG, Harris AL, Gatter KC, and Pezzella F. 2005. A quantitative analysis of lymphatic vessels in human breast cancer, based on LYVE-1 immunoreactivity. Br J Cancer 93:1168-1174. 10.1038/sj.bjc.6602844

Koukourakis MI, Giatromanolaki A, Sivridis E, Simopoulos C, Gatter KC, Harris AL, and Jackson DG. 2005. LYVE1 immunohistochemical assessment of lymphangiogenesis in endometrial and lung cancer. J Clin Pathol 58:202-206. 10.1136/jcp.2004.019174

Kropotova ES, Zinov'eva OL, Zyrianova AF, Choinzonov EL, Afanas'ev SG, Cherdyntseva NV, Beresten SF, Oparina N, and Mashkova TD. 2013. [Expression of genes involved in retinoic acid biosynthesis in human gastric cancer]. Mol Biol (Mosk) 47:317-330. 10.7868/s0026898413020079

Langfelder P, and Horvath S. 2008. WGCNA: an R package for weighted correlation network analysis. BMC Bioinformatics 9:559. 10.1186/1471-2105-9-559

Langfelder P, Luo R, Oldham MC, and Horvath S. 2011a. Is My Network Module Preserved and Reproducible? Plos Computational Biology 7.

Langfelder P, Luo R, Oldham MC, and Horvath S. 2011b. Is my network module preserved and reproducible? PLoS Comput Biol 7:e1001057. 10.1371/journal.pcbi.1001057

Lee JS, Kim SH, Lee S, Kang JH, Lee SH, Cheong JH, and Kim SY. 2019. Gastric cancer depends on aldehyde dehydrogenase 3A1 for fatty acid oxidation. Sci Rep 9:16313. 10.1038/s41598-019-52814-1

Lemeshow DWHS. 2000. Applied Logistic Regression, 2nd Ed.

Li C, Zeng X, Yu H, Gu Y, and Zhang W. 2018a. Identification of hub genes with diagnostic values in pancreatic cancer by bioinformatics analyses and supervised learning methods. World J Surg Oncol 16:223. 10.1186/s12957-018-1519-y

Li F, Du H, Li S, and Liu J. 2018b. The Association Between Metabolic Syndrome and Gastric Cancer in Chinese. Front Oncol 8:326. 10.3389/fonc.2018.00326

Li X, Yu W, Liang C, Xu Y, Zhang M, Ding X, and Cai X. 2020. INHBA is a prognostic predictor for patients with colon adenocarcinoma. BMC Cancer 20:305. 10.1186/s12885-020-06743-2

Lin H, Hong YG, Zhou JD, Gao XH, Yuan PH, Xin C, Huang ZP, Zhang W, Hao LQ, and Hou KZ. 2020a. LncRNA INHBA-AS1 promotes colorectal cancer cell proliferation by sponging miR-422a to increase AKT1 axis. Eur Rev Med Pharmacol Sci 24:9940-9948. 10.26355/eurrev_202010_23206

Lin TA, Wu TS, Li YJ, Yang CN, Illescas Ralda MM, and Chang HH. 2020b. Role and Mechanism of LIF in Oral Squamous Cell Carcinoma Progression. J Clin Med 9. 10.3390/jcm9020295

Liu D, Zhou D, Sun Y, Zhu J, Ghoneim D, Wu C, Yao Q, Gamazon ER, Cox NJ, and Wu L. 2020. A TranscriptomeWide Association Study Identifies Candidate Susceptibility Genes for Pancreatic Cancer Risk. Cancer Res 80:4346-4354. 10.1158/0008-5472.CAN-20-1353

Peer) reviewing PDF | (2020:08:51875:2:1:NEW 19 Nov 2020) 
552 Liu X, Li G, Ai L, Ye Q, Yu T, and Yang B. 2019a. Prognostic value of ATAD3 gene cluster expression in

553

554

555

556

557

558

559

560

561

562

563

564

565

566

567

568

569

570

571

572

573

574

575

576

577

578

579

580

581

582

583

584

585

586

587

588

589

590

591

592 hepatocellular carcinoma. Oncol Lett 18:1304-1310. 10.3892/ol.2019.10454

Liu Z, Li M, Hua Q, Li Y, and Wang G. 2019b. Identification of an eight-lncRNA prognostic model for breast cancer using WGCNA network analysis and a Cox-proportional hazards model based on L1-penalized estimation. International journal of molecular medicine 44:1333-1343.

Lou Y, Tian GY, Song Y, Liu YL, Chen YD, Shi JP, and Yang J. 2017. Characterization of transcriptional modules related to fibrosing-NAFLD progression. Sci Rep 7:4748. 10.1038/s41598-017-05044-2

Mendez-Hernandez A, Gallegos-Arreola MP, Moreno-Macias H, Espinosa Fematt J, and Perez-Morales R. 2017. LEP rs7799039, LEPR rs1137101, and ADIPOQ rs2241766 and 1501299 Polymorphisms Are Associated With Obesity and Chemotherapy Response in Mexican Women With Breast Cancer. Clin Breast Cancer 17:453462. 10.1016/j.clbc.2017.03.010

Miao YD, Wang JT, Ma XP, Yang Y, and Mi D. 2020. Identification prognosis-associated immune genes in colon adenocarcinoma. Biosci Rep. 10.1042/BSR20201734

Miyamoto Y, Schirripa M, Suenaga M, Cao S, Zhang W, Okazaki S, Berger MD, Matsusaka S, Yang D, Ning Y, Baba H, Loupakis F, Lonardi S, Pietrantonio F, Borelli B, Cremolini C, Yamaguchi T, and Lenz HJ. 2020. A polymorphism in the cachexia-associated gene INHBA predicts efficacy of regorafenib in patients with refractory metastatic colorectal cancer. PLoS One 15:e0239439. 10.1371/journal.pone.0239439

Mouta Carreira C, Nasser SM, di Tomaso E, Padera TP, Boucher Y, Tomarev SI, and Jain RK. 2001. LYVE-1 is not restricted to the lymph vessels: expression in normal liver blood sinusoids and down-regulation in human liver cancer and cirrhosis. Cancer Res 61:8079-8084.

Neidlin M, Dimitrakopoulou S, and Alexopoulos LG. 2019. Multi-tissue network analysis for drug prioritization in knee osteoarthritis. Sci Rep 9:15176. 10.1038/s41598-019-51627-6

Ngiam KY, and Khor IW. 2019. Big data and machine learning algorithms for health-care delivery. Lancet Oncol 20:e262-e273. 10.1016/S1470-2045(19)30149-4

Nimptsch K, Song M, Aleksandrova K, Katsoulis M, Freisling H, Jenab M, Gunter MJ, Tsilidis KK, Weiderpass E, Bueno-De-Mesquita HB, Chong DQ, Jensen MK, Wu C, Overvad K, Kuhn T, Barrdahl M, Melander O, Jirstrom K, Peeters PH, Sieri S, Panico S, Cross AJ, Riboli E, Van Guelpen B, Myte R, Huerta JM, RodriguezBarranco M, Quiros JR, Dorronsoro M, Tjonneland A, Olsen A, Travis R, Boutron-Ruault MC, Carbonnel F, Severi G, Bonet C, Palli D, Janke J, Lee YA, Boeing H, Giovannucci EL, Ogino S, Fuchs CS, Rimm E, Wu K, Chan AT, and Pischon T. 2017. Genetic variation in the ADIPOQ gene, adiponectin concentrations and risk of colorectal cancer: a Mendelian Randomization analysis using data from three large cohort studies. Eur J Epidemiol 32:419-430. 10.1007/s10654-017-0262-y

Nowak FV. 2018. Porf-2 = Arhgap39 = Vilse: A Pivotal Role in Neurodevelopment, Learning and Memory. eNeuro 5. 10.1523/ENEURO.0082-18.2018

Obeidat M, Nie Y, Chen V, Shannon CP, Andiappan AK, Lee B, Rotzschke O, Castaldi PJ, Hersh CP, Fishbane N, Ng RT, McManus B, Miller BE, Rennard S, Pare PD, and Sin DD. 2017. Network-based analysis reveals novel gene signatures in peripheral blood of patients with chronic obstructive pulmonary disease. Respir Res 18:72. 10.1186/s12931-017-0558-1

Oh SC, Sohn BH, Cheong JH, Kim SB, Lee JE, Park KC, Lee SH, Park JL, Park YY, Lee HS, Jang HJ, Park ES, Kim SC, Heo J, Chu IS, Jang YJ, Mok YJ, Jung W, Kim BH, Kim A, Cho JY, Lim JY, Hayashi Y, Song S, Elimova E, Estralla JS, Lee JH, Bhutani MS, Lu Y, Liu W, Lee J, Kang WK, Kim S, Noh SH, Mills GB, 
593

594

595

596

597

598

599

600

601

602

603

604

605

606

607

608

609

610

611

612

613

614

615

616

617

618

619

620

621

622

623

624

625

626

627

628

629

630

631

632

633

Kim SY, Ajani JA, and Lee JS. 2018. Clinical and genomic landscape of gastric cancer with a mesenchymal phenotype. Nat Commun 9:1777. 10.1038/s41467-018-04179-8

Oshima T, Yoshihara K, Aoyama T, Hasegawa S, Sato T, Yamamoto N, Akito N, Shiozawa M, Yoshikawa T, Numata K, Rino Y, Kunisaki C, Tanaka K, Akaike M, Imada T, and Masuda M. 2014. Relation of INHBA gene expression to outcomes in gastric cancer after curative surgery. Anticancer Res 34:2303-2309.

Ozmen F, Ozmen MM, Ozdemir E, Moran M, Seckin S, Guc D, Karaagaoglu E, and Kansu E. 2011. Relationship between LYVE-1, VEGFR-3 and CD44 gene expressions and lymphatic metastasis in gastric cancer. World J Gastroenterol 17:3220-3228. 10.3748/wjg.v17.i27.3220

Parida S, Siddharth S, and Sharma D. 2019. Adiponectin, Obesity, and Cancer: Clash of the Bigwigs in Health and Disease. Int J Mol Sci 20. 10.3390/ijms20102519

Peng G, Dan W, Jun W, Junjun Y, Tong R, Baoli Z, and Yang X. 2015. Transcriptome profiling of the cancer and adjacent nontumor tissues from cervical squamous cell carcinoma patients by RNA sequencing. Tumour Biol 36:3309-3317. 10.1007/s13277-014-2963-0

Pormohammad A, Mohtavinejad N, Gholizadeh P, Dabiri H, Salimi Chirani A, Hashemi A, and Nasiri MJ. 2018. Global estimate of gastric cancer in Helicobacter pylori-infected population: A systematic review and metaanalysis. J Cell Physiol. 10.1002/jcp.27114

Raimondi A, Nichetti F, Peverelli G, Di Bartolomeo M, De Braud F, and Pietrantonio F. 2018. Genomic markers of resistance to targeted treatments in gastric cancer: potential new treatment strategies. Pharmacogenomics 19:1047-1068. 10.2217/pgs-2018-0077

Ritchie ME, Phipson B, Wu D, Hu Y, Law CW, Shi W, and Smyth GK. 2015. limma powers differential expression analyses for RNA-sequencing and microarray studies. Nucleic Acids Res 43:e47. 10.1093/nar/gkv007

Robin X, Turck N, Hainard A, Tiberti N, Lisacek F, Sanchez JC, and Muller M. 2011. pROC: an open-source package for $\mathrm{R}$ and $\mathrm{S}+$ to analyze and compare ROC curves. BMC Bioinformatics 12:77. 10.1186/1471-2105-12-77

Sapkota S, Horiguchi K, Tosaka M, Yamada S, and Yamada M. 2017. Whole-Exome Sequencing Study of Thyrotropin-Secreting Pituitary Adenomas. J Clin Endocrinol Metab 102:566-575. 10.1210/jc.2016-2261

Sarver AE, Sarver AL, Thayanithy V, and Subramanian S. 2015. Identification, by systematic RNA sequencing, of novel candidate biomarkers and therapeutic targets in human soft tissue tumors. Lab Invest 95:1077-1088. 10.1038/labinvest.2015.80

Seeruttun SR, Cheung WY, Wang W, Fang C, Liu ZM, Li JQ, Wu T, Wang J, Liang C, and Zhou ZW. 2019. Identification of molecular biomarkers for the diagnosis of gastric cancer and lymph-node metastasis. Gastroenterol Rep (Oxf) 7:57-66. 10.1093/gastro/goy023

Shahraz A, Kopatz J, Mathy R, Kappler J, Winter D, Kapoor S, Schutza V, Scheper T, Gieselmann V, and Neumann H. 2015. Anti-inflammatory activity of low molecular weight polysialic acid on human macrophages. Sci Rep 5:16800. 10.1038/srep16800

Smyth EC, Verheij M, Allum W, Cunningham D, Cervantes A, Arnold D, and Committee EG. 2016. Gastric cancer: ESMO Clinical Practice Guidelines for diagnosis, treatment and follow-up. Ann Oncol 27:v38-v49. 10.1093/annonc/mdw350

Stefan Fritsch FG, Marvin N. Wright, Marc Suling, Sebastian M. Mueller. 2019. neuralnet: Training of Neural Networks.

Stratford JK, Bentrem DJ, Anderson JM, Fan C, Volmar KA, Marron JS, Routh ED, Caskey LS, Samuel JC, Der CJ, Thorne LB, Calvo BF, Kim HJ, Talamonti MS, Iacobuzio-Donahue CA, Hollingsworth MA, Perou CM, and

PeerJ reviewing PDF | (2020:08:51875:2:1:NEW 19 Nov 2020) 
634

635

636

637

638

639

640

641

642

643

644

645

646

647

648

649

650

651

652

653

654

655

656

657

658

659

660

661

662

663

664

665

666

667

668

669

670

671

672

673

674

Yeh JJ. 2010. A six-gene signature predicts survival of patients with localized pancreatic ductal adenocarcinoma. PLoS Med 7:e1000307. 10.1371/journal.pmed.1000307

Sun YL, Zhang Y, Guo YC, Yang ZH, and Xu YC. 2020. A Prognostic Model Based on the Immune-related Genes in Colon Adenocarcinoma. Int J Med Sci 17:1879-1896. 10.7150/ijms.45813

Takeno A, Takemasa I, Doki Y, Yamasaki M, Miyata H, Takiguchi S, Fujiwara Y, Matsubara K, and Monden M. 2008. Integrative approach for differentially overexpressed genes in gastric cancer by combining large-scale gene expression profiling and network analysis. Br J Cancer 99:1307-1315. 10.1038/sj.bjc.6604682

Tan X, Wang GB, Tang Y, Bai J, and Ye L. 2017. Association of ADIPOQ and ADIPOR variants with risk of colorectal cancer: A meta-analysis. J Huazhong Univ Sci Technolog Med Sci 37:161-171. 10.1007/s11596017-1710-3

Tan Y, Lin K, Zhao Y, Wu Q, Chen D, Wang J, Liang Y, Li J, Hu J, Wang H, Liu Y, Zhang S, He W, Huang Q, Hu X, Yao Z, Liang B, Liao W, and Shi M. 2018. Adipocytes fuel gastric cancer omental metastasis via PITPNC1-mediated fatty acid metabolic reprogramming. Theranostics 8:5452-5468. 10.7150/thno.28219

Teng Y, Lang L, and Shay C. 2019. ATAD3A on the Path to Cancer. Adv Exp Med Biol 1134:259-269. 10.1007/978-3030-12668-1_14

Teng Y, Ren X, Li H, Shull A, Kim J, and Cowell JK. 2016. Mitochondrial ATAD3A combines with GRP78 to regulate the WASF3 metastasis-promoting protein. Oncogene 35:333-343. 10.1038/onc.2015.86

Waldum HL, Sagatun L, and Mjones P. 2017. Gastrin and Gastric Cancer. Front Endocrinol (Lausanne) 8:1. $10.3389 /$ fendo.2017.00001

Wang J, Ni Z, Duan Z, Wang G, and Li F. 2014. Altered expression of hypoxia-inducible factor-1alpha (HIF-1alpha) and its regulatory genes in gastric cancer tissues. PLoS One 9:e99835. 10.1371/journal.pone.0099835

Wang Q, Wen YG, Li DP, Xia J, Zhou CZ, Yan DW, Tang HM, and Peng ZH. 2012. Upregulated INHBA expression is associated with poor survival in gastric cancer. Med Oncol 29:77-83. 10.1007/s12032-010-9766-y

Wen F, Huang J, Lu X, Huang W, Wang Y, Bai Y, Ruan S, Gu S, Chen X, and Shu P. 2020. Identification and prognostic value of metabolism-related genes in gastric cancer. Aging (Albany NY) 12:17647-17661. 10.18632/aging.103838

Wu S, Liu X, He J, Wang H, Luo Y, Gong W, Li Y, Huang Y, Zhong L, and Zhao Y. 2019. A Dual Targeting Magnetic Nanoparticle for Human Cancer Detection. Nanoscale Res Lett 14:228. 10.1186/s11671-019-3049-0

Xiao B, Kuang Z, Zhang W, Hang J, Chen L, Lei T, He Y, Deng C, Li W, Lu J, Qu J, Zhou Q, Hao W, Sun Z, and Li L. 2019. Glutamate Ionotropic Receptor Kainate Type Subunit 3 (GRIK3) promotes epithelial-mesenchymal transition in breast cancer cells by regulating SPDEF/CDH1 signaling. Mol Carcinog 58:1314-1323. $10.1002 / \mathrm{mc} .23014$

Xu GJ, Shah AA, Li MZ, Xu Q, Rosen A, Casciola-Rosen L, and Elledge SJ. 2016. Systematic autoantigen analysis identifies a distinct subtype of scleroderma with coincident cancer. Proc Natl Acad Sci U S A 113:E7526E7534. 10.1073/pnas.1615990113

Yoon H, and Kim N. 2015. Diagnosis and management of high risk group for gastric cancer. Gut Liver 9:5-17. $10.5009 / \mathrm{gn} 114118$

Zeng X, Li C, Li Y, Yu H, Fu P, Hong HG, and Zhang W. 2019. A network-based variable selection approach for identification of modules and biomarker genes associated with end-stage kidney disease. Nephrology (Carlton). 10.1111/nep.13655

Zhang B, and Horvath S. 2005. A general framework for weighted gene co-expression network analysis. Stat Appl

Peer) reviewing PDF | (2020:08:51875:2:1:NEW 19 Nov 2020) 
675

676

677

678

679

680

681

682

683

684

685

686
Genet Mol Biol 4:Article17. 10.2202/1544-6115.1128

Zhang C, Liang Y, Ma MH, Wu KZ, and Dai DQ. 2019. KRT15, INHBA, MATN3, and AGT are aberrantly methylated and differentially expressed in gastric cancer and associated with prognosis. Pathol Res Pract 215:893-899. 10.1016/j.prp.2019.01.034

Zhang X, Yang JJ, Kim YS, Kim KY, Ahn WS, and Yang S. 2010. An 8-gene signature, including methylated and down-regulated glutathione peroxidase 3, of gastric cancer. Int J Oncol 36:405-414.

Zhao C, Li Y, Chen G, Wang F, Shen Z, and Zhou R. 2017. Overexpression of miR-15b-5p promotes gastric cancer metastasis by regulating PAQR3. Oncol Rep 38:352-358. 10.3892/or.2017.5673

Zhou XG, Huang XL, Liang SY, Tang SM, Wu SK, Huang TT, Mo ZN, and Wang QY. 2018. Identifying miRNA and gene modules of colon cancer associated with pathological stage by weighted gene co-expression network analysis. Onco Targets Ther 11:2815-2830. 10.2147/OTT.S163891 
Figure 1

Figure 1. Flow diagram of the study

data collection, analysis, and hub gene selection and validation. 


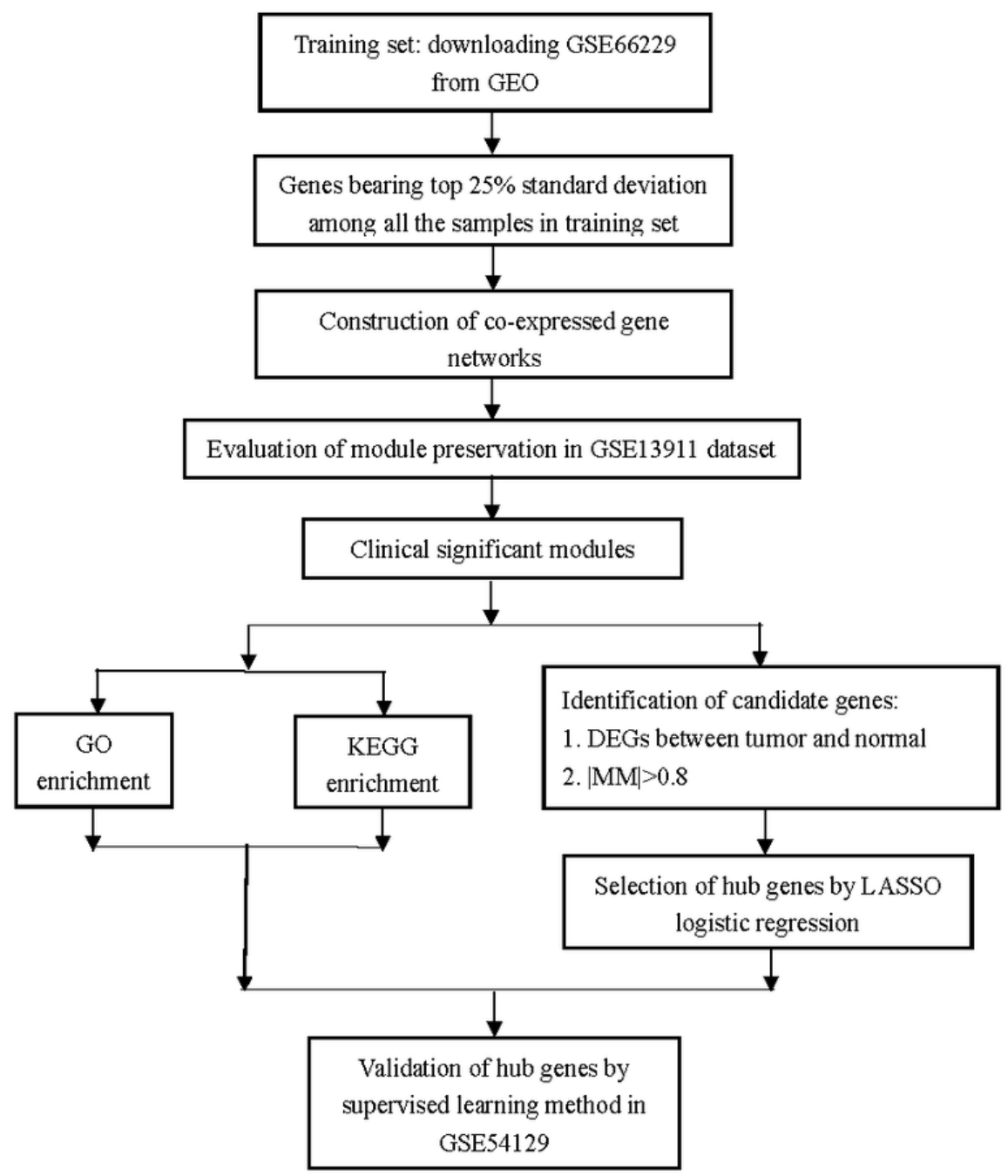


Figure 2

Figure 2. Determination of the soft-thresholding power in the weighted gene coexpression network analysis in the training set.

(A) Screening soft-thresholding powers. (B) Analysis of the mean connectivity for various softthresholding powers. (C) Histogram of the connectivity distribution with the soft-thresholding powers set at 4. (D) Checking the scale-free topology with the soft-thresholding powers set at 4. 
A

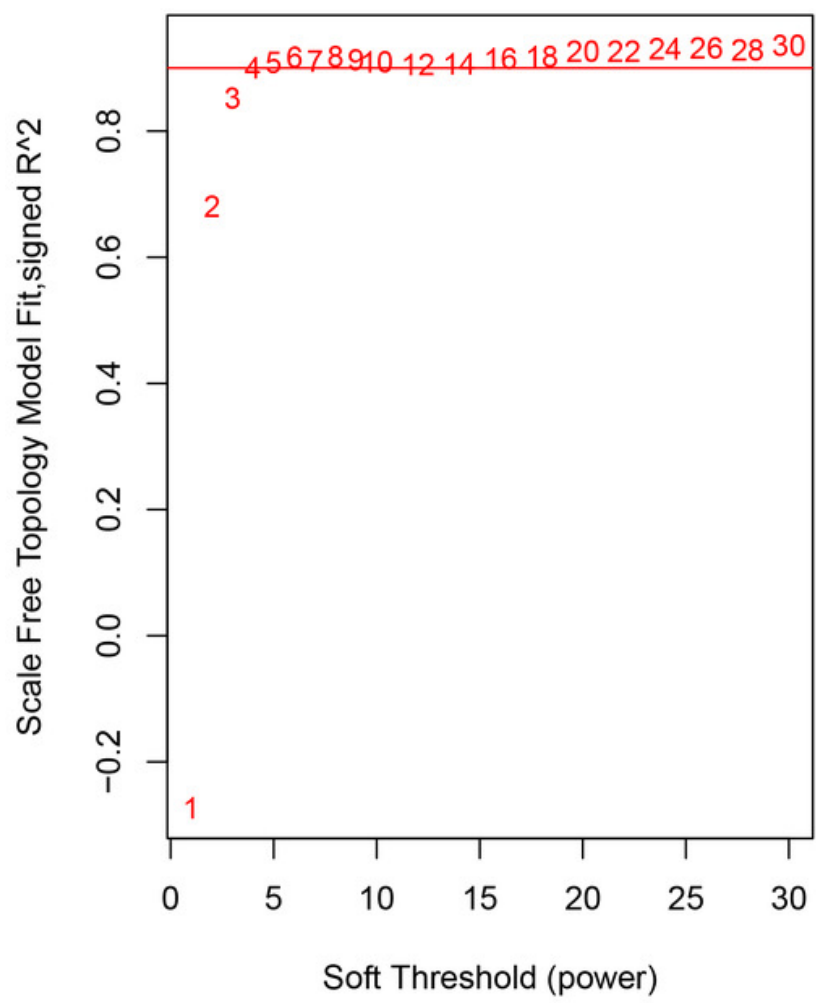

C

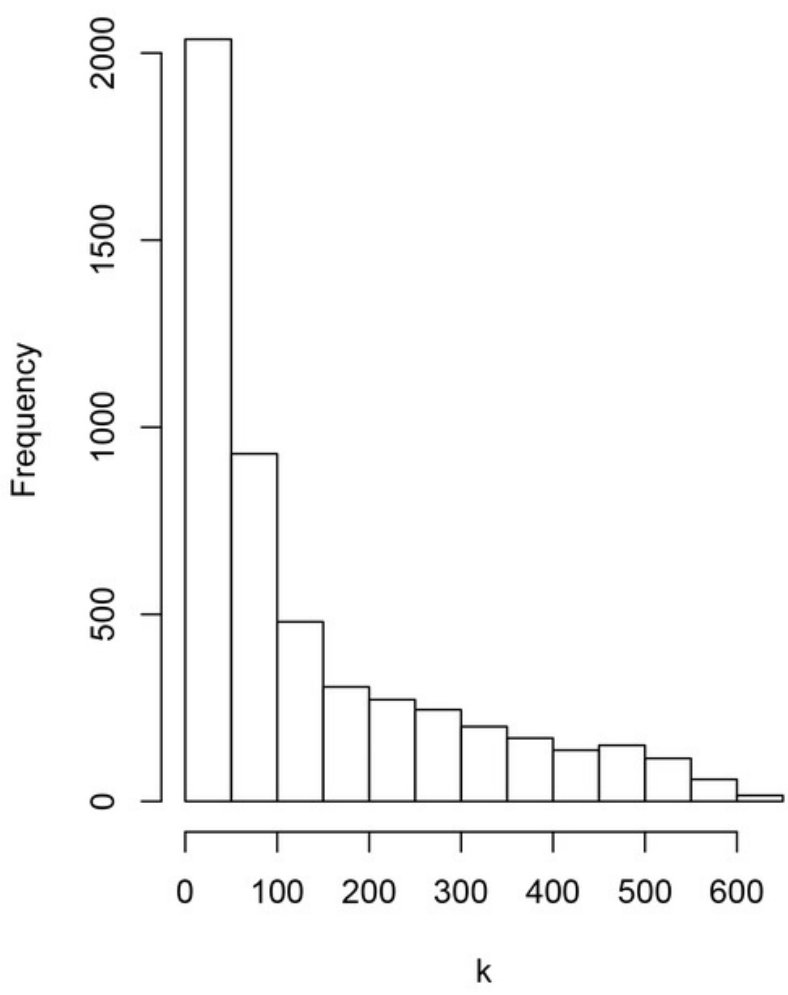

B

Mean connectivity

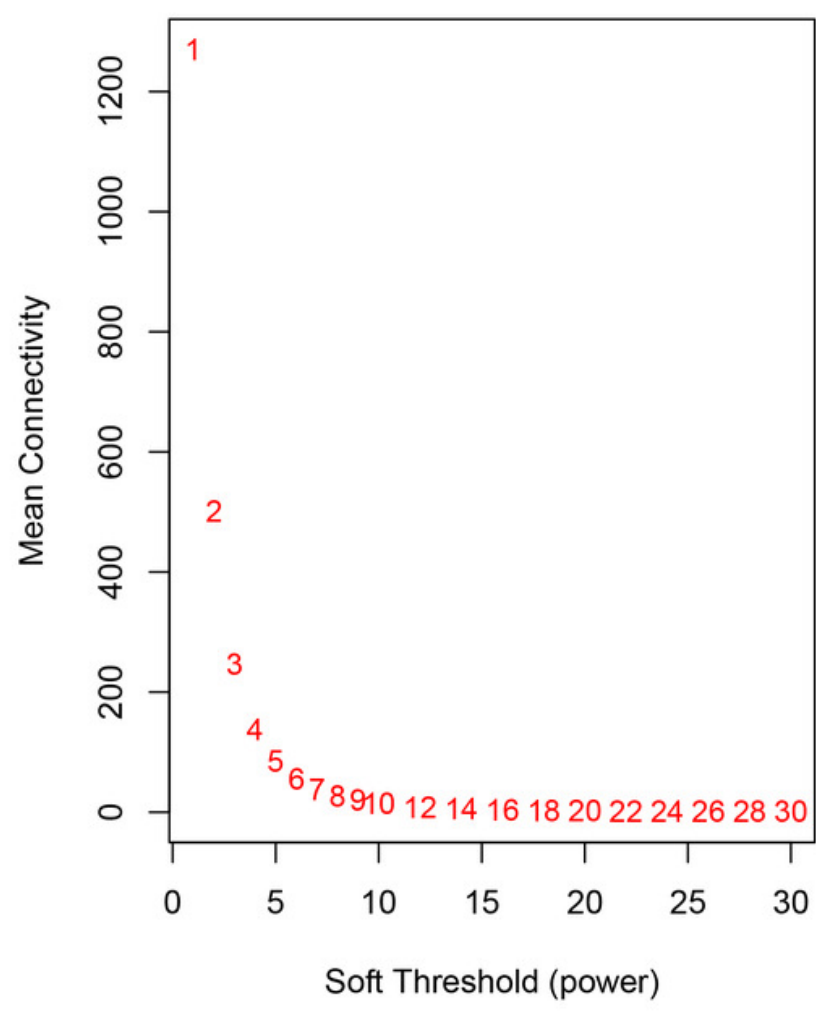

D Check scale free topology

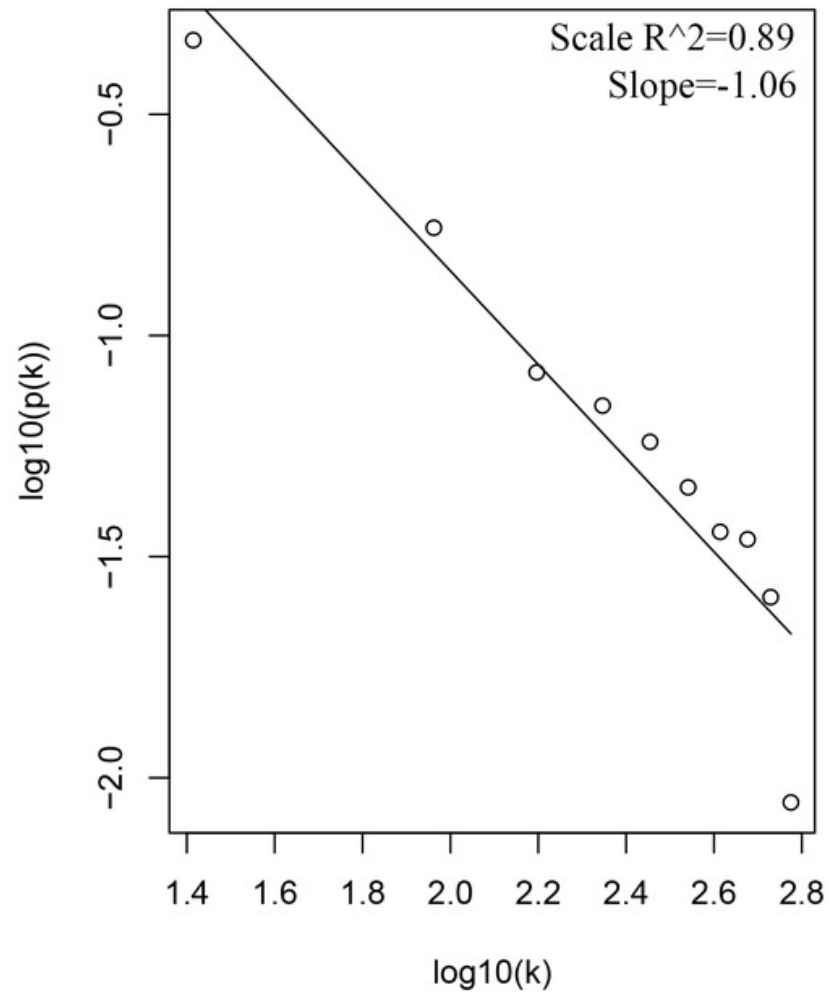


Figure 3

Figure 3. Clustering dendrograms of the 5115 genes in the training set.

(A) Clustering of the module eigengenes to identify the merged modules. Upon setting the threshold at $0.25,15$ modules were merged into 12 modules (B) Co-expression module of the training set. 
A

Clustering of module eigengenes

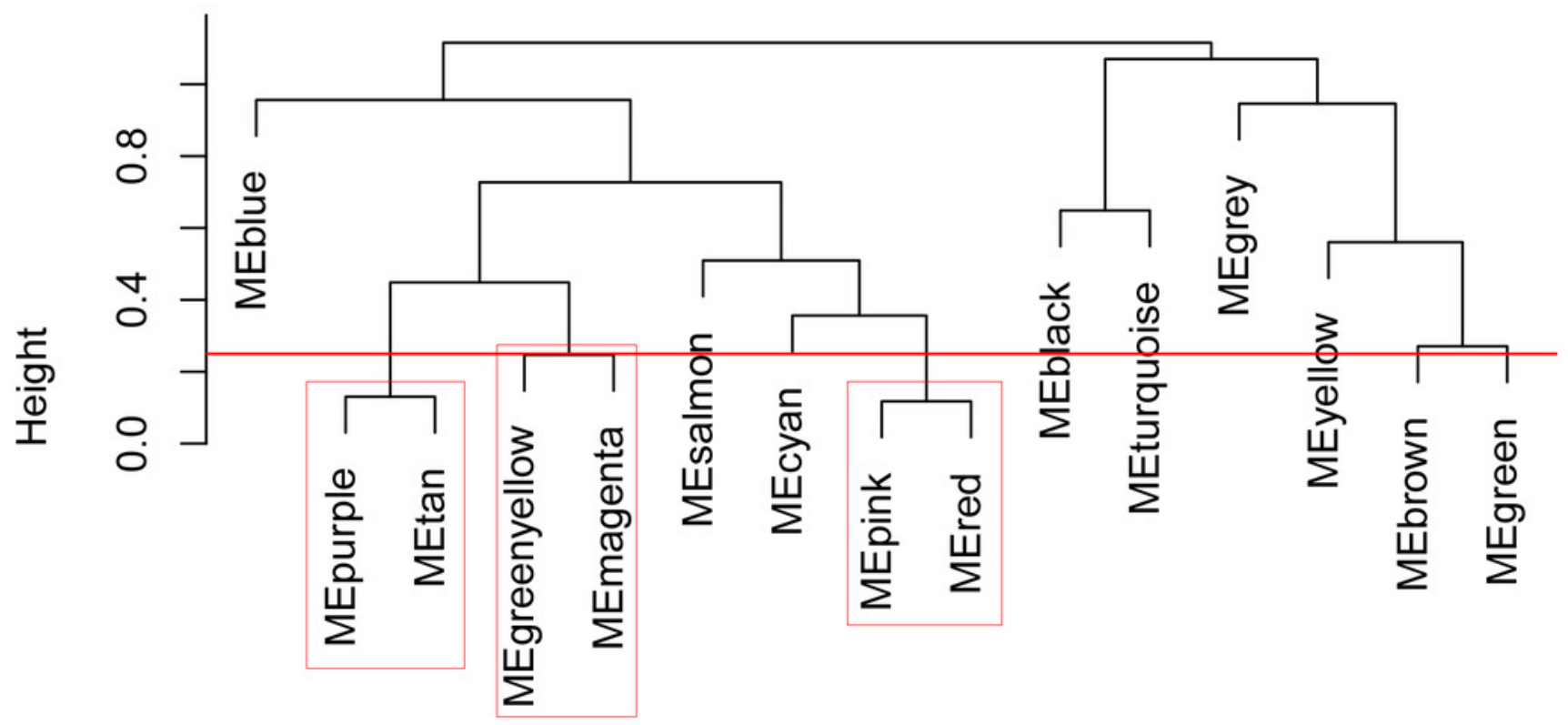

B

Cluster Dendrogram

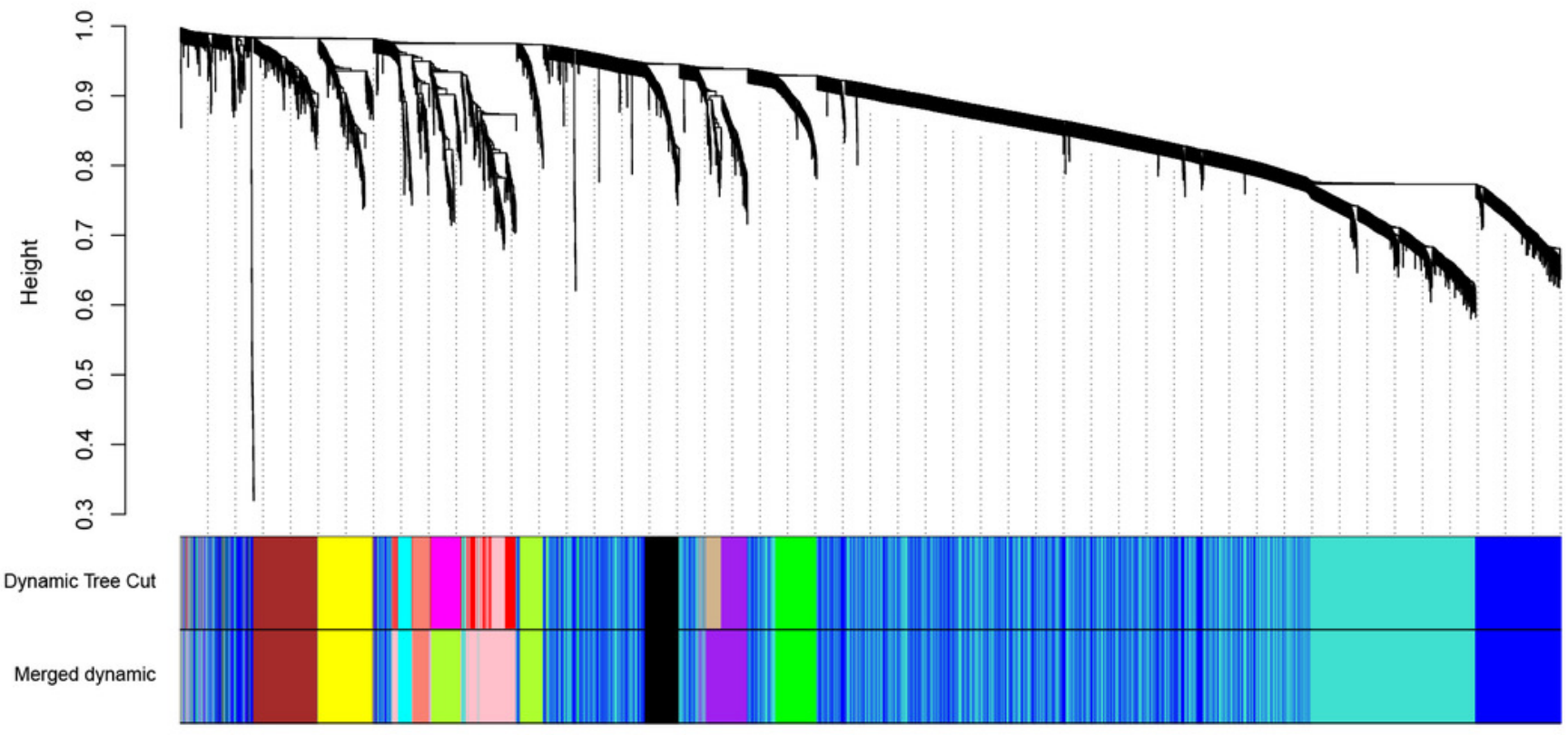


Figure 4

Figure 4. Evaluation of module preservation.

The $x$ - and $y$-axes present module size and preservation median rank (A) as well as preservation Z summary (B), respectively.
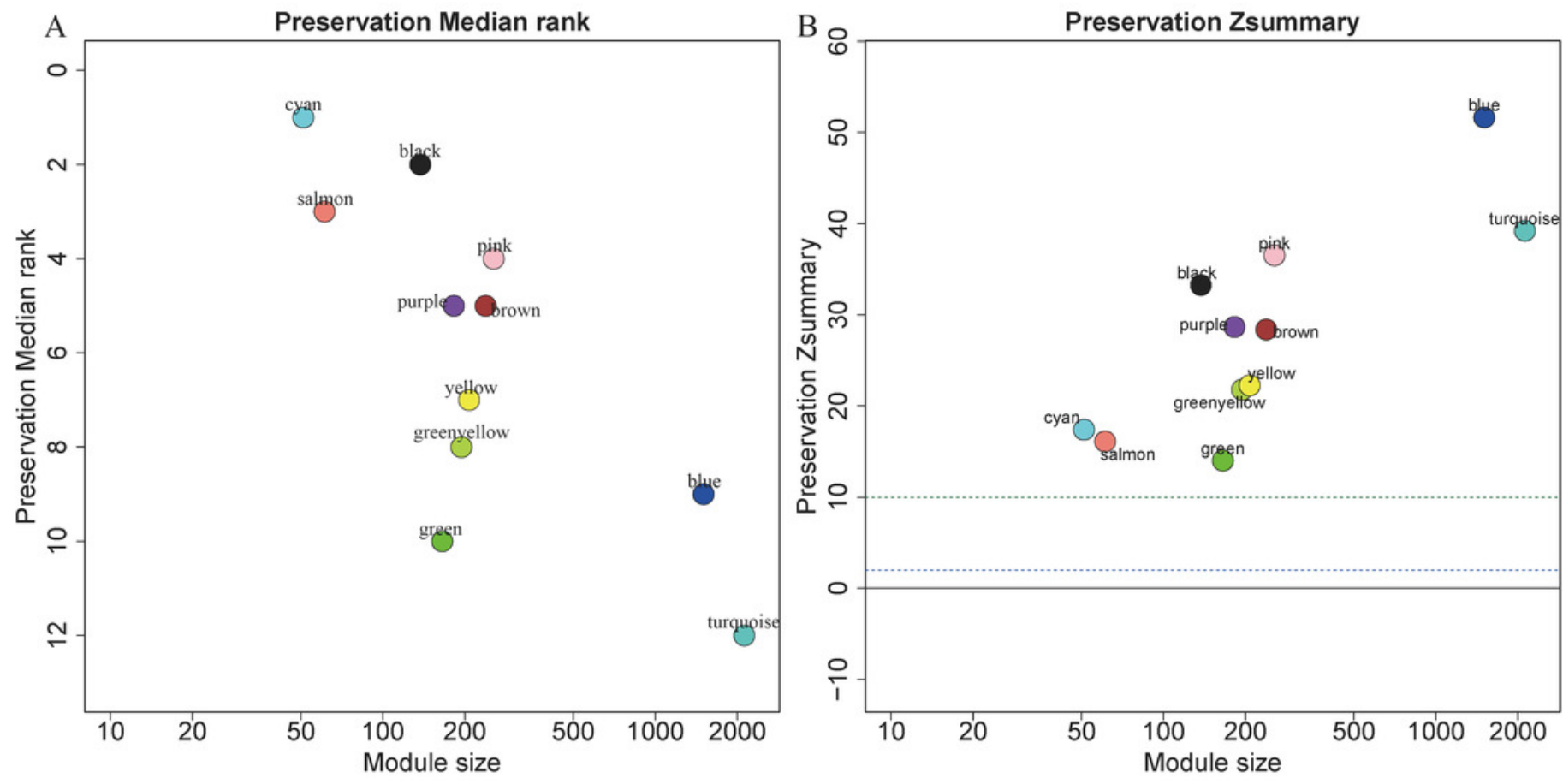


\section{Figure 5}

Figure 5. Identification of clinically significant modules.

$\log _{10}$ transformation of the $P$-value in the logistic regression between the MEs and clinical trait. The height of bars represents the $\log _{10}$ transformation of the $P$-value, and modules with $\log _{10}$ transformation of the $P$-value greater than 10 were considered to be closely correlated with tissue types.

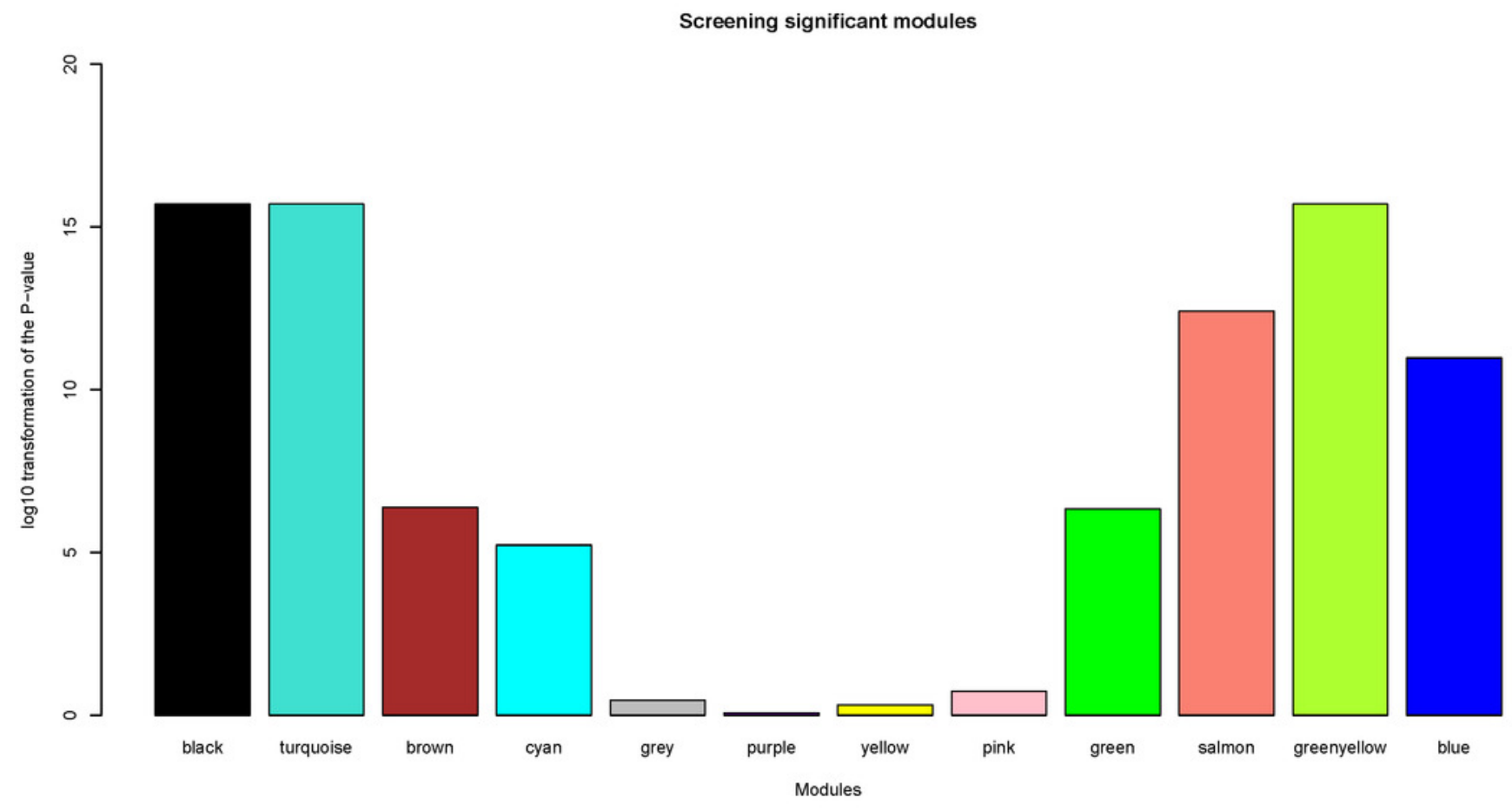




\section{Figure 6}

Figure 6. The bubble plot of gene ontology terms in the A) blue, B) black, C) turquoise, D) greenyellow and E) salmon modules.

The $z$-score is assigned to the $x$-axis and the negative logarithm of the $p$-value to the $y$-axis, as in the barplot (the higher the more significant). The area of the displayed circles is proportional to the number of genes assigned to the terms. Herein, only the meaningful enriched GO terms were presented, and the most three sigificant GO terms' lables were displayed. 

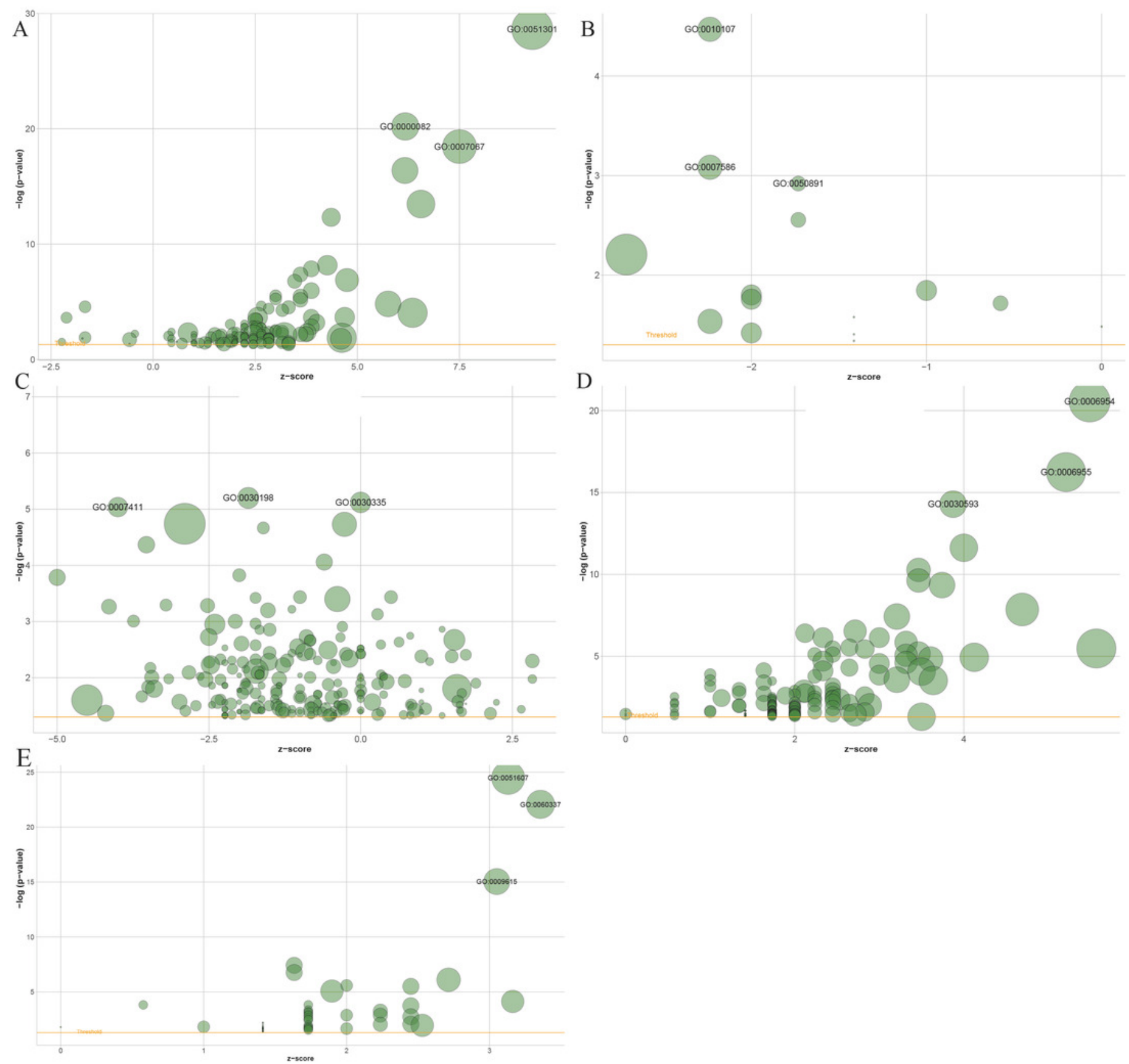


\section{Figure 7}

Figure 7. KEGG pathway enrichment analyses of the (A) blue, (B) black, C) turquoise, D) greenyellow and E) salmon modules.

The negative logarithm of the $p$-value is assigned to the $x$-axis and the term of each pathway to the $y$-axis. The size of the bubble shows the numbers of the genes enriched in each pathway, while the colors indicate the enrichment significance (from blue to red designated as less to high significance). 


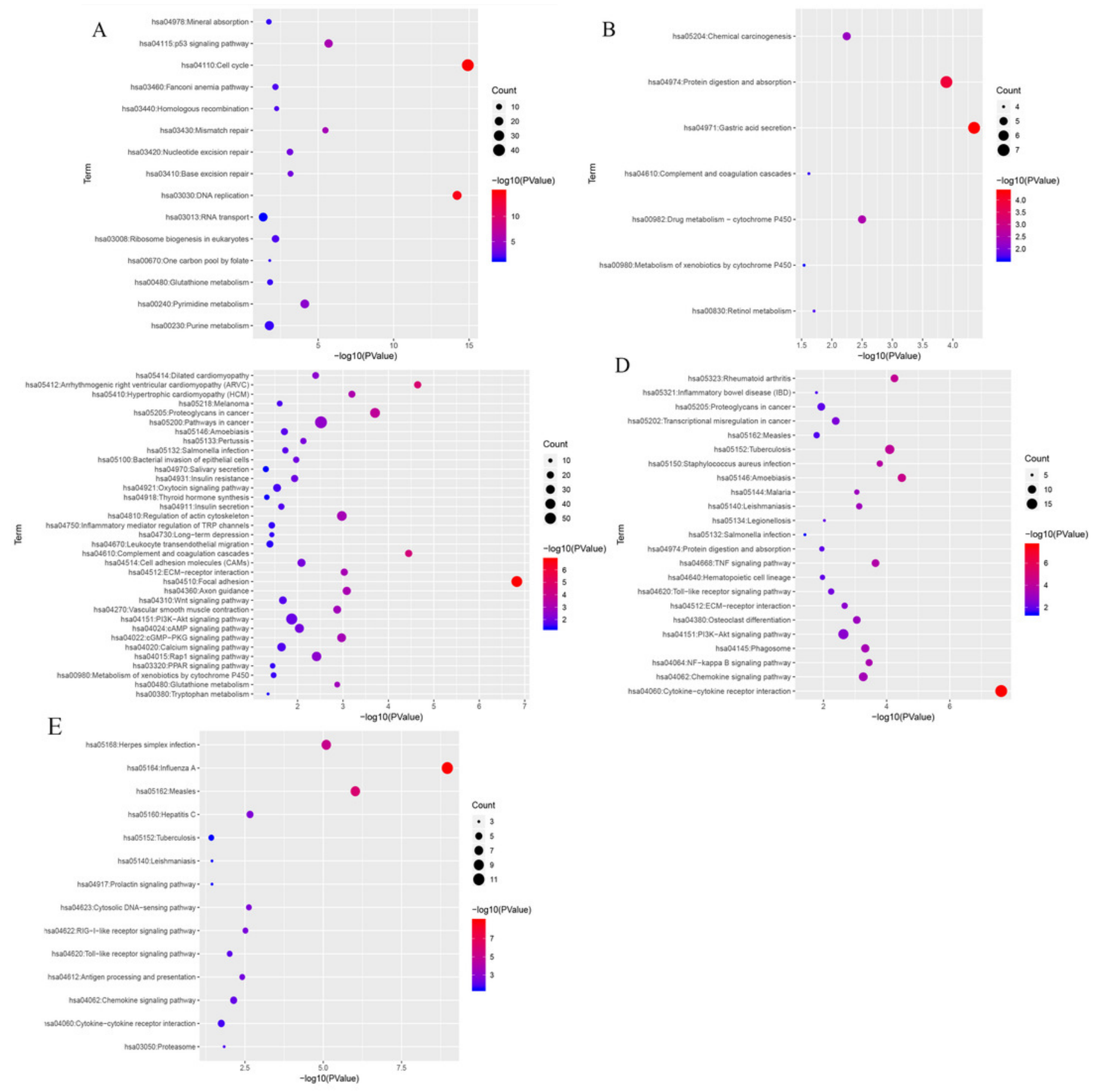




\section{Figure 8}

Figure 8. Heatmaps showing the expressopms pf 11 hub genes between the gastric cancer patients and normal controls in training set.

The $\mathrm{x}$ - and $\mathrm{y}$-axes present the samples and genes, respectively. In the $\mathrm{x}$-axis, pink and green represent the gastric cancer and normal samples, respectively. The scale bar from blue to red represented low to high expressions of each gene in each sample.

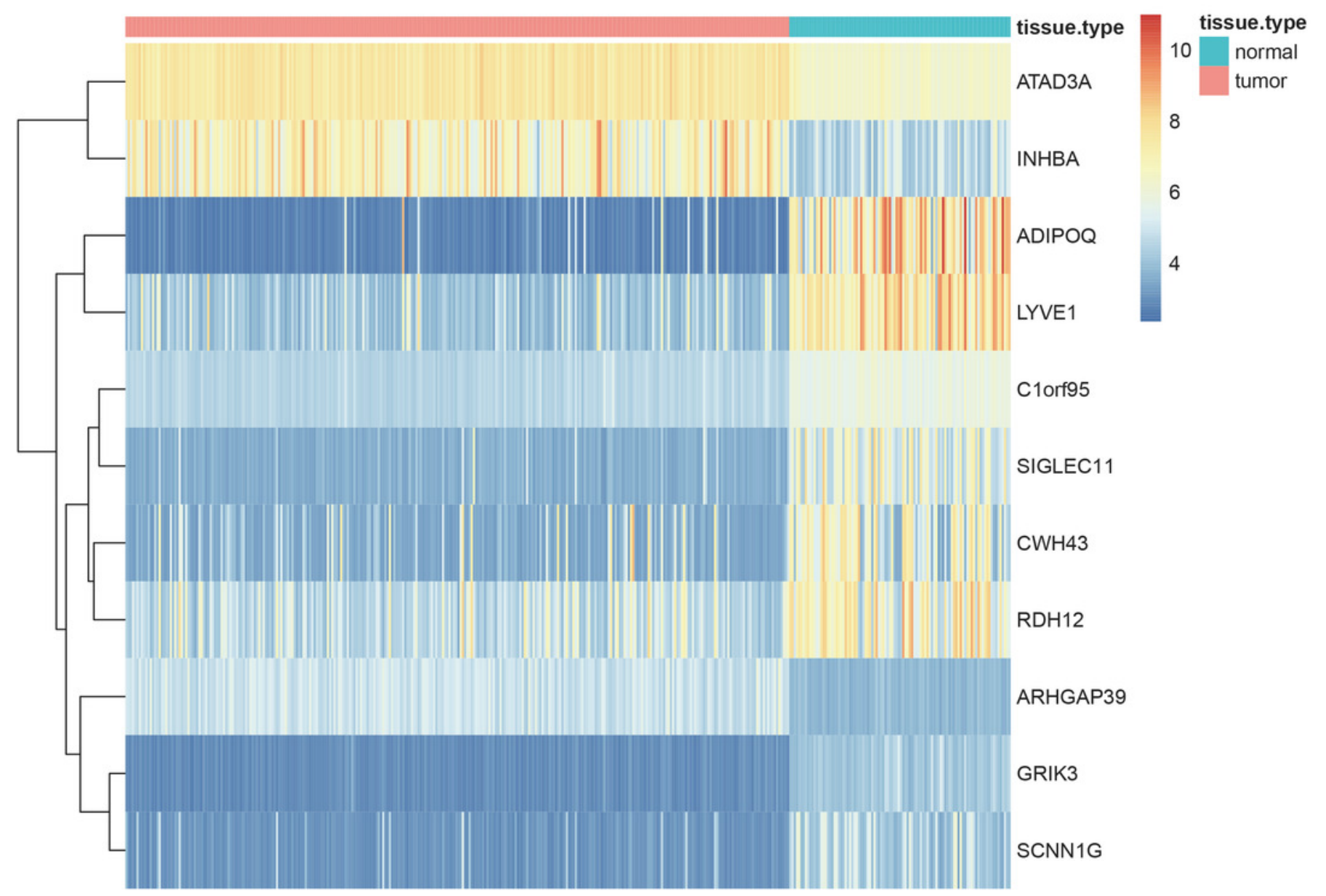


Figure 9

Figure 9. Validation results.

ROC curve of the classifier predicted by these 11 hub genes in the testing set of A) gastric cancer, B) colorectal cancer and C) pancreatic cancer .
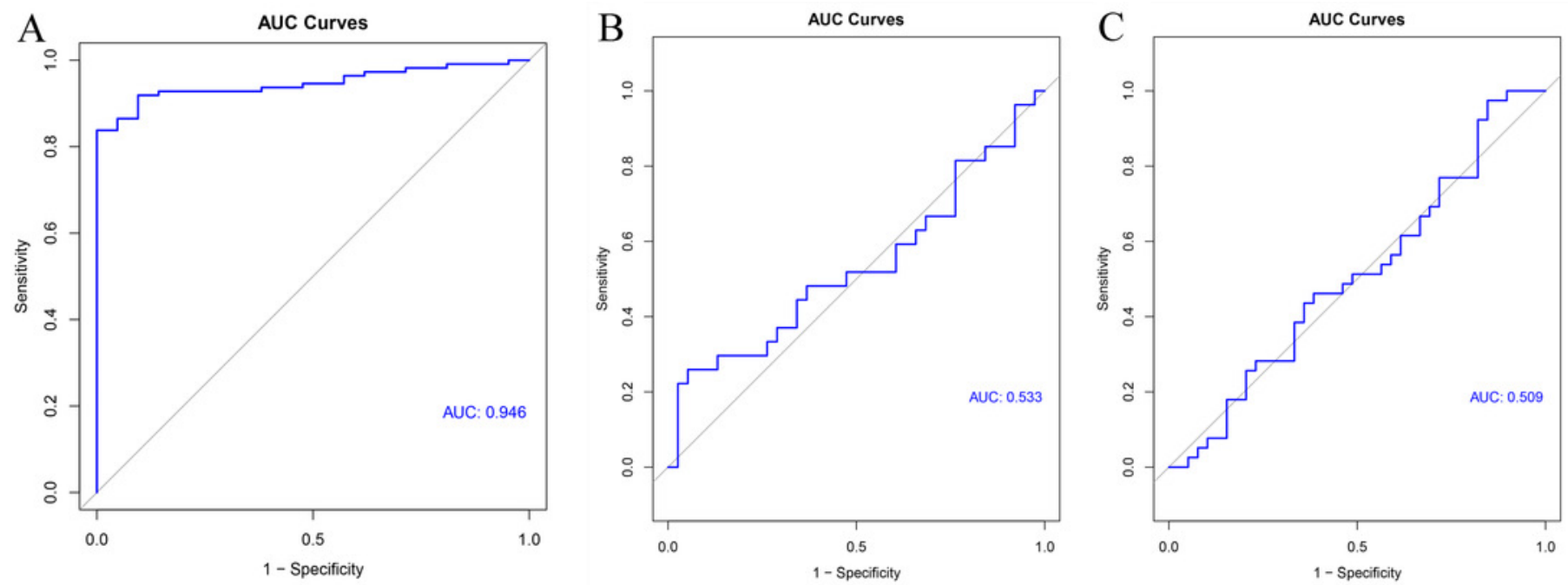


\section{Table $\mathbf{1}$ (on next page)}

Table 1. LASSO regression results.

Genes selected by the LASSO logistic regression, with the estimated coefficients and odds ratio. 
1 Table 1. LASSO regression results. Genes selected by the LASSO logistic regression, with the 2 estimated coefficients and odds ratio.

\begin{tabular}{lll}
\hline Gene & Coefficient & Odds Ratio \\
\hline ADIPOQ & -0.16241431554 & 0.8500889265 \\
ARHGAP39 & 1.14238882470 & 3.134246595 \\
ATAD3A & 0.85913784838 & 2.361124169 \\
C1orf95 & -1.99447221881 & 0.1360854586 \\
CWH43 & -0.05325279770 & 0.9481402945 \\
GRIK3 & -4.02900881511 & 0.01779195633 \\
INHBA & 0.19110414846 & 1.210585526 \\
LYVE1 & -0.01065353474 & 0.9894030132 \\
RDH12 & -0.09270793497 & 0.9114596669 \\
SCNN1G & -0.02709362847 & 0.9732701115 \\
SIGLEC11 & -0.17337880264 & 0.84081905 \\
\hline
\end{tabular}

3 